\title{
Nano-targeted induction of dual ferroptotic mechanisms eradicates high-risk neuroblastoma
}

\author{
Behrouz Hassannia, ${ }^{1,2}$ Bartosz Wiernicki, ${ }^{1,2}$ Irina Ingold, ${ }^{3}$ Feng Qu, ${ }^{4}$ Simon Van Herck, ${ }^{5}$ Yulia Y. Tyurina, ${ }^{4}$ Hülya Bayır, ${ }^{4}$ \\ Behnaz A. Abhari, ${ }^{6}$ Jose Pedro Friedmann Angeli,, ${ }^{7}$ Sze Men Choi, ${ }^{1,2}$ Eline Meul, ${ }^{1,2}$ Karen Heyninck, ${ }^{8}$ Ken Declerck, ${ }^{9}$ \\ Chandra Sekhar Chirumamilla, ${ }^{9}$ Maija Lahtela-Kakkonen, ${ }^{10}$ Guy Van Camp, ${ }^{11}$ Dmitri V. Krysko, ${ }^{1,2}$ Paul G. Ekert, ${ }^{12}$ Simone Fulda, ${ }^{6,13}$ \\ Bruno G. De Geest, ${ }^{5}$ Marcus Conrad, ${ }^{3}$ Valerian E. Kagan, ${ }^{4}$ Wim Vanden Berghe, ${ }^{8,9}$ Peter Vandenabeele, ${ }^{1,2,14}$ and Tom Vanden Berghe ${ }^{1,2}$ \\ ${ }^{1} V I B$ Center for Inflammation Research, Ghent, Belgium. ²Department of Biomedical Molecular Biology, Chent University, Ghent, Belgium. ${ }^{3}$ Helmholtz Zentrum München, Institute of Developmental Genetics, \\ Neuherberg, Germany. . ${ }^{4}$ Center for Free Radical and Antioxidant Health, University of Pittsburgh, Pittsburgh, Pennsylvania, USA. ${ }^{5}$ Department of Pharmaceutics, Ghent University, Ghent, Belgium. ${ }^{6}$ Institute \\ for Experimental Cancer Research in Pediatrics, Goethe-University, Frankfurt, Germany. ${ }^{7}$ Rudolf Virchow Center for Experimental Biomedicine, University of Würzburg, Würzburg, Germany. ${ }^{8}$ Laboratory \\ of Eukaryotic Gene Expression and Signal Transduction, Department of Physiology, Ghent University, Chent, Belgium. 'ªboratory of Protein Chemistry, Proteomics and Epigenetic Signalling (PPES), \\ Department of Biomedical Sciences, University of Antwerp, Wilrijk, Belgium. ${ }^{10}$ School of Pharmacy, Pharmaceutical Chemistry, University of Eastern Finland, Kuopio, Finland. "Center of Medical Cenetics, \\ Department of Biomedical Sciences, University of Antwerp, Antwerp, Belgium. ${ }^{12}$ Cancer Research, Murdoch Children's Research Institute, Royal Children's Hospital, Parkville, Victoria, Australia. ${ }^{13}$ German \\ Cancer Consortium (DKTK) and German Cancer Research Center (DKFZ), Heidelberg, Germany. ${ }^{14}$ Methusalem Program, Ghent University, Ghent, Belgium.
}

High-risk neuroblastoma is a devastating malignancy with very limited therapeutic options. Here, we identify withaferin A (WA) as a natural ferroptosis-inducing agent in neuroblastoma, which acts through a novel double-edged mechanism. WA dose-dependently either activates the nuclear factor-like 2 pathway through targeting of Kelch-like ECH-associated protein 1 (noncanonical ferroptosis induction) or inactivates glutathione peroxidase 4 (canonical ferroptosis induction). Noncanonical ferroptosis induction is characterized by an increase in intracellular labile Fe(II) upon excessive activation of heme oxygenase-1, which is sufficient to induce ferroptosis. This double-edged mechanism might explain the superior efficacy of WA as compared with etoposide or cisplatin in killing a heterogeneous panel of high-risk neuroblastoma cells, and in suppressing the growth and relapse rate of neuroblastoma xenografts. Nano-targeting of WA allows systemic application and suppressed tumor growth due to an enhanced accumulation at the tumor site. Collectively, our data propose a novel therapeutic strategy to efficiently kill cancer cells by ferroptosis.

\section{Introduction}

Neuroblastoma is a neural crest-derived malignancy that accounts for approximately $15 \%$ of pediatric cancer death (1). Based on biological and clinical features such as age at diagnosis, stage of disease (L1, L2, M, and Ms), and molecular markers such as MYCN oncogene amplification, patients are divided into low-, intermediate-, and high-risk groups (2). Despite extensive multi-agent therapy, a considerable number of high-risk-group patients, determined with MYCN amplification and/or metastatic disease stage (stage $\mathrm{M})$, have a poor clinical outcome and do not respond to therapy or relapse after treatment (1). Therefore, there is an urgent need to identify novel drugs or treatment strategies for these patients.

Cancer cells often acquire genetic mutations and abnormal gene expression in extrinsic and intrinsic apoptotic pathways (3). Therefore, alternative caspase-independent cell death modalities such as necroptosis $(4,5)$ and ferroptosis $(6)$ could provide alternative treatment paradigms to eradicate apoptosis-resistant cancer cells (7). Necroptosis, the best-characterized form of regulated necrosis, is mediated by the concerted action of receptor-interacting protein

Conflict of interest: The authors have declared that no conflict of interest exists Submitted: December 5, 2017; Accepted: April 27, 2018.

Reference information: J Clin Invest. 2018;128(8):3341-3355.

https://doi.org/10.1172/JCI99032 kinase 3 (RIPK3) and mixed-lineage kinase domain-like (MLKL) (8). Ferroptosis is triggered by inactivation of the lipid repair enzyme glutathione peroxidase 4 (GPX4), which is followed by accumulation of toxic lipid peroxides leading to cell death $(6,9)$. The redox-active iron contributes to the execution of ferroptosis by promoting formation of phospholipid peroxyl radicals (10), which characterizes ferroptosis as an iron-catalyzed form of necrosis. Because neoplastic cells have higher levels of iron than nonmalignant cells (11), exploiting ferroptosis has proven an alternative and highly efficient way to kill therapy-resistant cancer cells (12).

Using a natural anticancer agent, withaferin A (WA), we now demonstrate that ferroptosis is a potent anticancer strategy to treat high-risk neuroblastoma. We further show that WA-induced ferroptosis in neuroblastoma involves a double-edged mechanism. On the one hand, WA drops the protein level and activity of GPX4, which resembles the canonical ferroptosis-inducing pathway. On the other hand, WA induces a novel noncanonical ferroptosis pathway by increasing the labile Fe(II) pool upon excessive activation of heme oxygenase 1 (HMOX1) through direct targeting of Kelch-like ECH-associated protein 1 (KEAP1), which is sufficient to induce lipid peroxidation. This double-edged targeting mechanism results in high efficacy of WA compared with etoposide and cisplatin in killing a heterogeneous panel of high-risk neuroblastoma cells, and in suppressing neuroblastoma xenograft 
growth and relapse. To increase the targeting of WA to the tumor, we generated WA-encapsulated nanoparticles. This nanomedicinal approach allowed systemic application and the effective suppression of tumor growth. Conclusively, the discovery of WAmediated ferroptosis by targeting of GPX 4 and/or enhancing of the labile $\mathrm{Fe}$ (II) pool through excessive HMOX1 activation might open new perspectives for the development of novel treatments in cell death-resistant cancer cells.

\section{Results}

WA kills etoposide-resistant neuroblastoma cells by inducing lipid peroxidation. Therapy resistance is a major issue in more than half of high-risk neuroblastoma patients. We and others have reported on the profound anticancer effect of WA (Supplemental Figure 1A; supplemental material available online with this article; https:// doi.org/10.1172/JCI99032DS1) especially on therapy-resistant cancer cells $(13,14)$. Therefore, we examined the sensitivity of a panel of high-risk neuroblastoma cell lines to WA, including $M Y C N$-amplified and nonamplified cells. We observed that WA is significantly more potent than the standard chemotherapeutics etoposide and cisplatin in killing these different neuroblastoma cell lines (Figure 1A and Supplemental Table 1).

We used 2 different neuroblastoma cell lines, one with MYCN amplification (IMR-32 cells) and one without (SK-N-SH cells), to further study the dose-response effects and mechanism of action of WA. The optimal cytotoxic dose was determined for both cell lines (Supplemental Figure 1, B and C). Notably, a structurally related inactive withanolide with a different epoxy stereo-orientation, withanone (WN), was not toxic to neuroblastoma cells (Supplemental Figure 1B), which is similar to what was previously observed in endothelial cells $(15,16)$. Although previous studies in a variety of cancer cell lines indicated WA-induced apoptosis (17), we observed that treatment with the caspase inhibitor Z-VAD-FMK did not block WA-induced cell death in IMR-32 or SK-N-SH cells (Supplemental Figure 1D). Time course analysis revealed no caspase activity or proteolytic processing of caspases during WA-induced cell death, while robust caspase activity was observed upon staurosporine treatment (Supplemental Figure 1, E and F). Live cell imaging revealed that WA-induced cell death occurred with a necrotic morphology including cell rounding, swelling, and plasma membrane rupture (Figure 1B). Lysosomal membrane permeabilization also occurred before plasma membrane rupture during cellular disintegration (Supplemental Videos 1 and 2), similar to what we have observed for other types of necrosis (18).

Since WA triggered caspase-independent cell death with necrotic morphology, we next investigated whether necroptosis, as a prototype form of regulated necrosis, was involved (7). Before exposure to WA, we pretreated the neuroblastoma cells with a panel of necroptosis inhibitors. With the exceptions of necrostatin-1 (Nec1) and butylated hydroxyanisole (BHA), none of the necroptosis inhibitors blocked WA-induced cell death in IMR-32 cells nor SK-N-SH cells (Supplemental Figure 1G and Supplemental Table 2). Nec1 and the lipophilic antioxidant BHA were previously shown to also inhibit ferroptosis $(19,20)$, although the precise mechanism in case of Nec1 remains elusive. Next, we pretreated the IMR-32 cells with a panel of ferroptosis inhibitors before exposing the cells to WA or erastin, a class I ferroptosis-inducing compound (FIN) that blocks the cystine/ glutamate antiporter (6). Interestingly, cell death induced by WA was blocked by this panel of ferroptosis inhibitors in both IMR-32 and SK-N-SH cells (Figure 1C, Supplemental Figure 1H, and Supplemental Table 3). Note that the class II FINs (1S,3R)-RSL3 (RSL3) and ML162 also effectively induced ferroptosis in a panel of high-risk neuroblastoma cell lines, while the class I FIN erastin was less effective in some cell lines (Supplemental Figure 1, H and I).

Considering that oxidative stress drives lipid peroxidation in ferroptosis, we analyzed both parameters after a WA challenge using the fluorochromes DHR123 and C11-BODIPY, respectively. We found that WA induces an oxidative burst (Supplemental Figure 1J) and an increase in lipid peroxidation in IMR-32 neuroblastoma cells (Figure 1D), which further supported a ferroptotic phenotype. Iron chelation using deferoxamine (DFO) blocked lipid peroxidation (Figure 1D), highlighting the catalytic role of iron in ferroptosis. Ferrostatin-1 (Fer1) was less effective than DFO in blocking WA-induced lipid peroxidation (Figure 1D) compared with erastin-induced lipid peroxidation (Supplemental Figure 1K). Lowering the dose of WA or increasing the dose of Fer1 did not further reduce lipid ROS to baseline (Supplemental Figure 1L). We observed the same incomplete protection against lipid hydroperoxide generation by Fer1 treatment using the class II FIN RSL3 (Supplemental Figure 1M). Lipid peroxidation could not be detected using a nonferroptotic trigger such as staurosporine (Supplemental Figure 1N). Conclusively we show that WA induces a necrotic-like cell death that presents all the hallmarks of ferroptosis.

WA acts as a natural class II ferroptosis-inducing agent in neuroblastoma. GPX4 inactivation is one of the key features of ferroptosis, which occurs either through a drop in glutathione (GSH) levels or by direct targeting of GPX4 (6). In contrast to the class I FIN erastin, we did not observe a significant drop in GSH levels in the neuroblastoma cells following WA treatment (Supplemental Figure 1O). This observation could indicate that the ferroptosisinducing action of WA may be attributed to binding and inactivation of GPX4, as previously shown for the class II FIN RSL3 (6). In line with our reasoning, we found a drop in the GPX4 protein level at both a cytotoxic high and medium dose of WA (Figure $1 \mathrm{E})$, resulting in a loss of GPX4 activity (Supplemental Figure 1P). Pull-down of WA from lysates of cells treated with biotinylated WA revealed GPX4 binding in the presence of a high dose of WA, but not in response to a cytotoxic medium dose of WA (Figure 1F). Accordingly, overexpression of WT GPX4 in IMR-32 cells delayed ferroptosis in response to a medium dose of WA, while no inhibition was observed in response to a high dose of WA (Supplemental Figure 1Q). Note that an inactivating mutation of the active site selenocysteine of GPX $4^{\mathrm{U} 46 \mathrm{~S}}$ abrogated the protective effect upon overexpression, which underscores the importance of its enzymatic activity to protect membranes against ferroptosis induced by a medium dose of WA.

WA preferentially alkylates nucleophilic sites such as protein thiols via Michael addition (21). To verify this mechanism of inactivation, we reconstituted WT GPX4 or a mutant in which all cysteines, except for the selenocysteine within its catalytic center, were mutated to serine (GPX $4^{\text {Allcys/Ser }}$ ) in tamoxifeninduced GPX4-deficient cells (22). While loss of GPX4 in response to a high dose of WA was evident in WT GPX4 and 
A

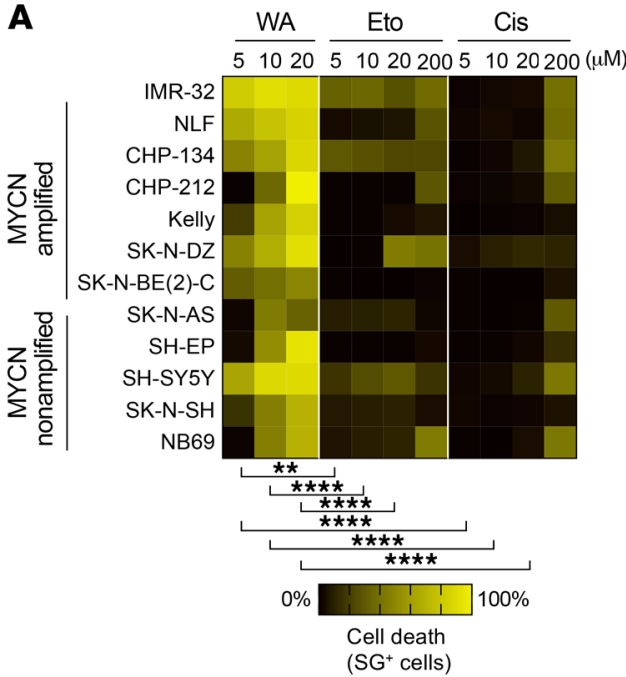

B

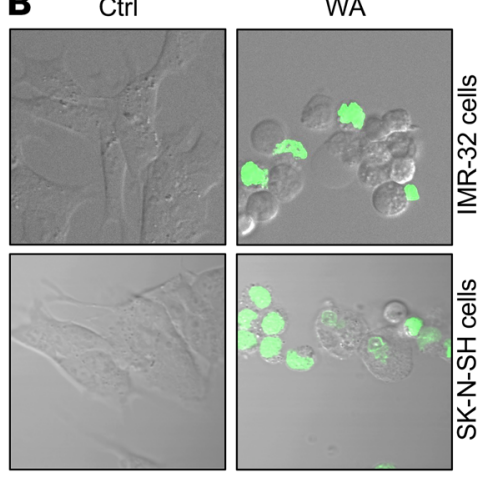

C

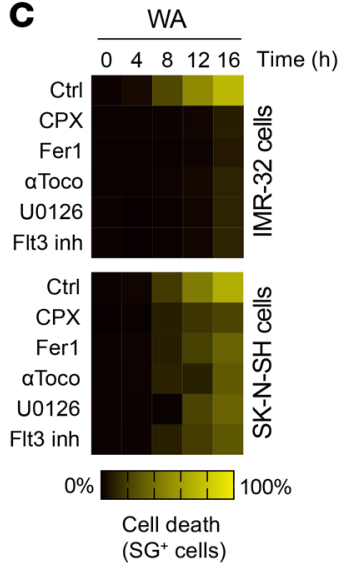

D

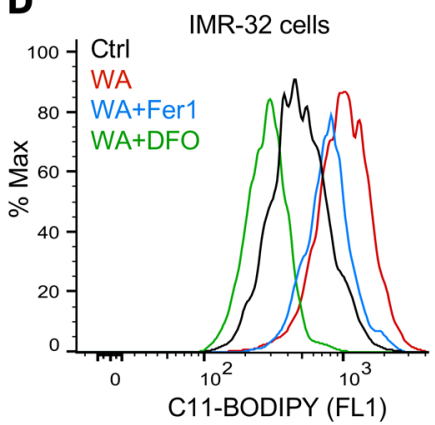

E

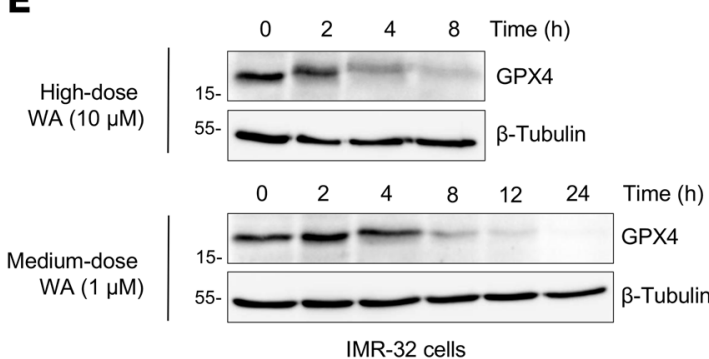

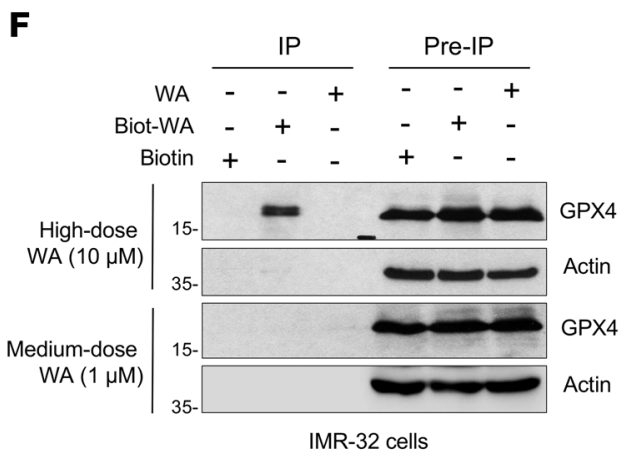

G

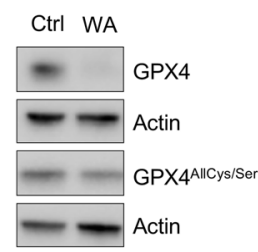

Reconstituted Gpx4 $4^{-1-}$ MEF cells

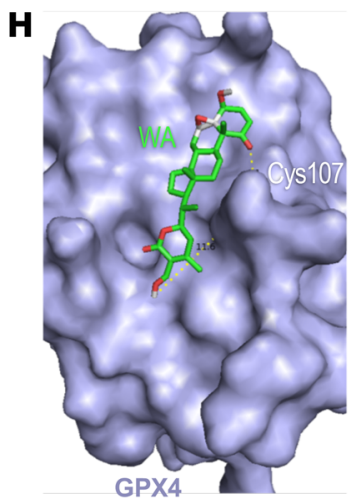

Figure 1. WA acts as a class II ferroptosis-inducing agent to kill etoposide- and cisplatin-resistant neuroblastoma cells. (A) Heatmap representing cell death sensitivity of various high-risk neuroblastoma cell lines after exposure to WA, etoposide (Eto), and cisplatin (Cis). See also Supplemental Table 1. SG, Sytox Green. (B) Snapshots from a live cell imaging of IMR-32 and SK-N-SH cells treated with WA. See also Supplemental Videos 1 and 2. Green fluorescent staining represents SytoxGreen. (C) Heatmap representing the cell death sensitivity of IMR-32 and SK-N-SH cells after exposure to $1 \mu \mathrm{M}$ or $10 \mu \mathrm{M}$ WA, respectively, in the absence or presence of different ferroptosis inhibitors, including the iron chelator ciclopirox olamine (CPX, 5 M), the lipid peroxidation inhibitor ferrostatin-1 (Fer1, $500 \mathrm{nM}$ ), the lipophilic antioxidant $\alpha$-tocopherol ( $\alpha$ Toco, $100 \mu \mathrm{M})$, and the kinase inhibitors U0126 $(10 \mu \mathrm{M})$ and Flt3 inhibitor (500nM). See also Supplemental Table 3. (D) Flow cytometric analysis of the lipid peroxidation sensor (C11-BODIPY-581/591 dye) on live-gated cells (SytoxBlue-negative cells) after treatment of IMR-32 cells with WA. (E) Western blot analysis revealing GPX4 and $\beta$-tubulin expression in IMR-32 cells treated with a high dose (10 $\mu$ M) and a medium dose $(1 \mu \mathrm{M})$ of WA. (F) Western blot analysis revealing GPX4 and actin after immunoprecipitation of biotin-WA in lysates of IMR-32 cells treated with a high dose (10 $\mu \mathrm{M})$ and a medium dose $(1 \mu \mathrm{M})$ of biotin-WA. (C) Western blot analysis revealing GPX4 expression in GPX4-depleted mouse embryonic fibroblasts (MEFs) reconstituted with GPX4 or GPX4 $4^{\text {All }(y / / 5 e r} 5$ hours after treatment with WA $(10 \mu \mathrm{M})$. (H) Molecular modeling simulation of WA docked on Cys107 of human GPX4 crystal structure (2OBI). See also Supplemental Table 4 and Supplemental Video 3. ${ }^{* *} P<0.01,{ }^{* * *} P<0.0001,2$-way ANOVA test (A).

GPX $4^{\mathrm{U} 46 \mathrm{C}}$ reconstituted cells, no drop was observed in the GPX $4^{\text {Allcys/Ser }}$ mutant (Figure $1 \mathrm{G}$ and Supplemental Figure 1R). Note that the structurally related inactive withanolide $\mathrm{WN}$ did not change GPX4 protein levels (Supplemental Figure 1R). This suggests that a high dose of WA interacts preferentially with cysteines, instead of selenocysteine.

Since DTT is a cysteine-rich thiol donor, we next evaluated whether cotreatment with DTT could compete for cysteine- 
A
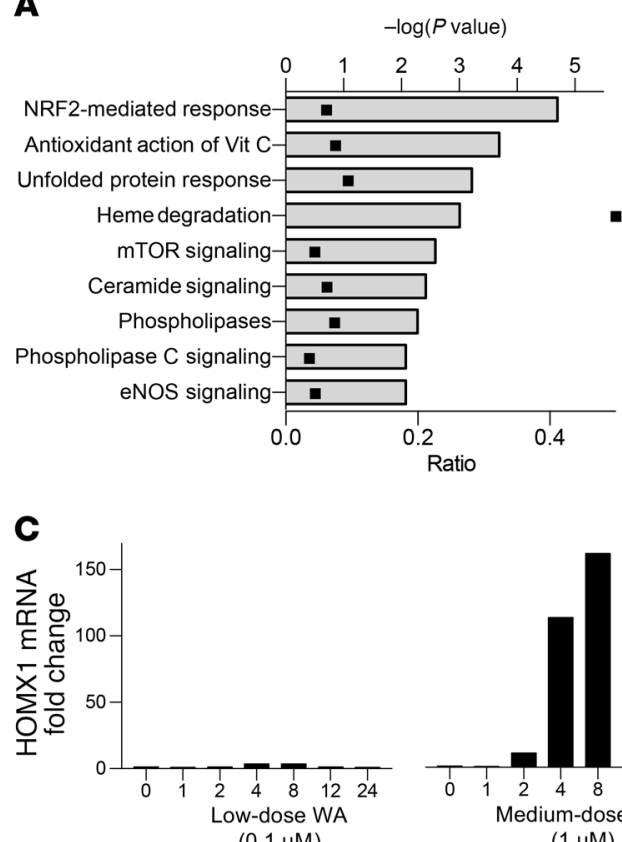

$(0.1 \mu \mathrm{M})$

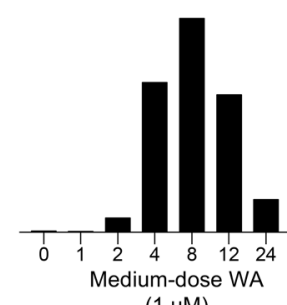

$(1 \mu \mathrm{M})$

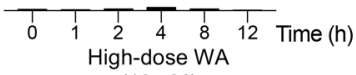

$(10 \mu \mathrm{M})$
Low-dose WA

$(0.1 \mu \mathrm{M})$

G

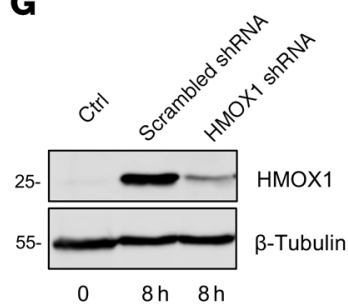

$\mathbf{K}$

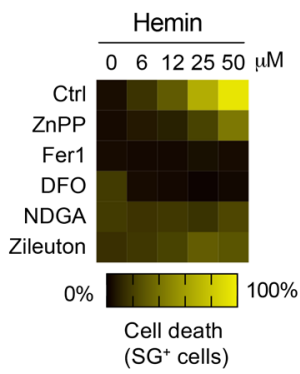

$\mathbf{L}$

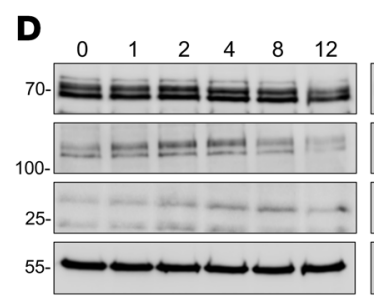

B

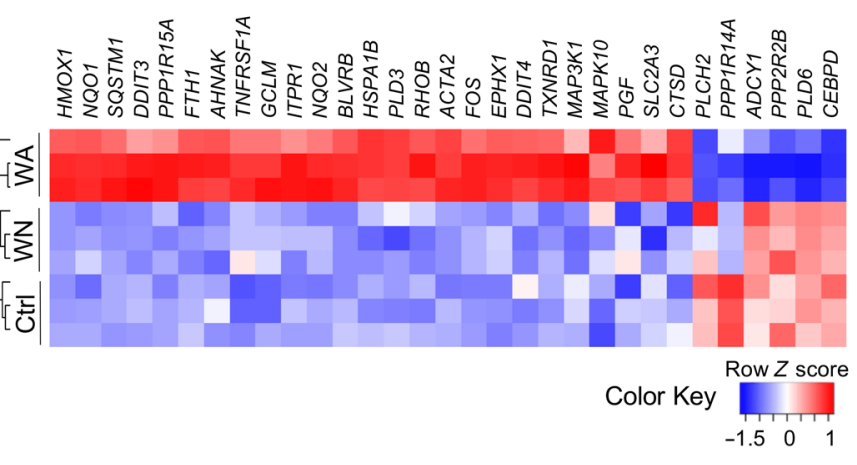

E

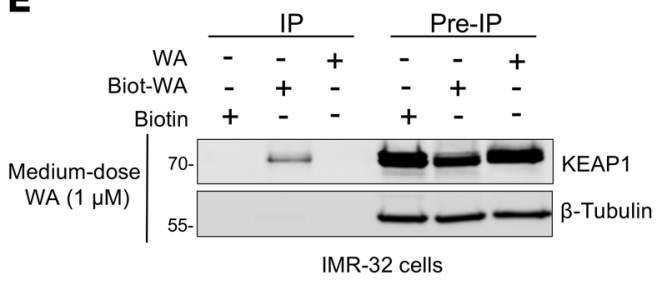

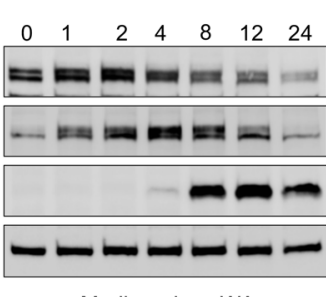

Medium-dose WA

$(1 \mu \mathrm{M})$
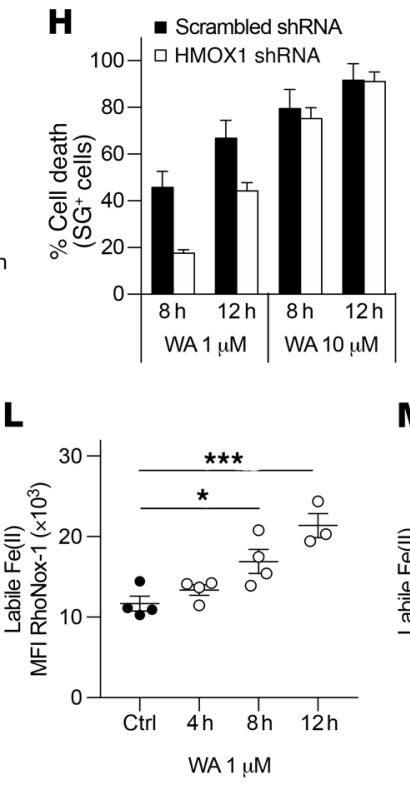

M

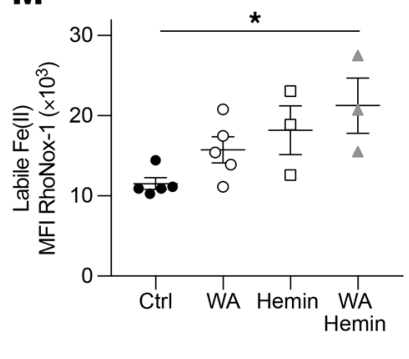

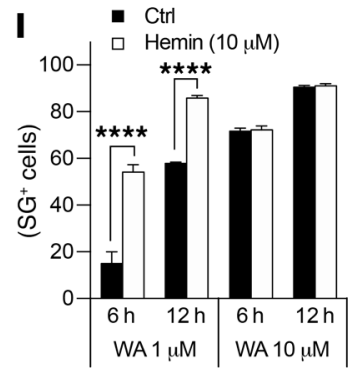

$\mathbf{F}$

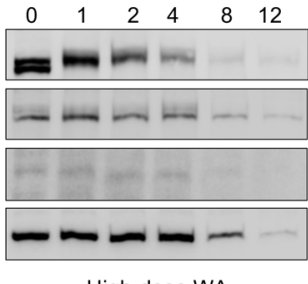

gh-dose WA

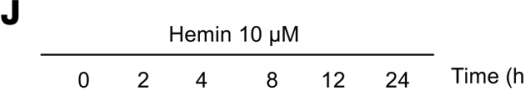

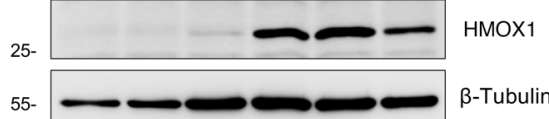

$\mathbf{N}$

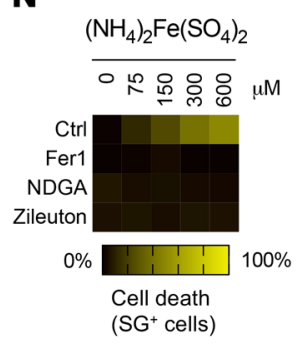


Figure 2. WA increases intracellular labile $\mathrm{Fe}$ (II) upon excessive activation of HMOX1, which is sufficient to induce ferroptosis. (A) IPA of genes differentially expressed after treatment with WA $(1 \mu \mathrm{M})$ in IMR-32 cells. (B) Gene expression profiles of Ctrl, WA-treated, and WN-treated IMR-32 cells. Color key legend represents Z score values. (C) Relative HMOX1 mRNA expression in IMR-32 cells after WA-treatment. (D) Western blot revealing KEAP1, NRF2, and HMOX1 in IMR-32 cells treated with WA. (E) Western blot revealing KEAP1 after immunoprecipitation of biotin-WA in lysates of IMR-32 cells treated with biotin-WA $(1 \mu \mathrm{M})$. (F) Percentage of cell death induced by WA in the presence/absence of HMOX1 inhibitor (ZnPP). (G) Western blot revealing HMOX1 in response to WA $(1 \mu \mathrm{M})$. (H) Percentage of cell death induced by WA upon RNAi-mediated knockdown of HMOX1. The results are representative of 2 independent experiments. (I) Percentage of cell death induced by WA in the presence/absence of hemin. (J) Western blot revealing HMOX1 in response to hemin $(10 \mu \mathrm{M})$. (K) Heatmap representing the sensitivity of IMR-32 cells in response to hemin in the presence of ferroptosis inhibitors. See also Supplemental Table 5. (L) Cellular levels of labile Fe(II), using RhoNox-1, in function of time in response to WA $(1 \mu \mathrm{M})$. (M) Cellular levels of labile Fe(II), using RhoNox-1, 8 hours after treatment with WA $(1 \mu \mathrm{M})$, hemin $(10 \mu \mathrm{M})$, or the combination. $\mathrm{MFI}$, mean fluorescence intensity. (N) Heatmap representing cell death sensitivity of IMR-32 cells in response to (NH4)2Fe(SO4)2 in the absence/ presence of ferroptosis inhibitors. See also Supplemental Table 6. The combined results of 2 or 3 independent experiments are shown for $\mathbf{F}, \mathbf{I}, \mathbf{K}$, and $\mathbf{N}$. Error bars represent SEM. ${ }^{*} P<0.05,{ }^{* *} P<0.001,{ }^{* * * *} P<0.0001$, 2-way ANOVA test (F, I, L,and $\mathbf{M})$. NDGA, nordihydroguaiaretic acid.

dependent WA binding to GPX4. As expected, upon addition of excess amounts of DTT to compete for GPX4 binding, interaction between biotinylated WA and GPX4 was completely lost, further supporting the alkylation of GPX4 by WA (Supplemental Figure 1S). To support our finding that WA covalently binds to GPX4 through cysteines, we performed molecular modeling to investigate the propensity of WA to bind solvent-exposed cysteines of human GPX4 (Protein Data Bank ID 2OBI). In silico modeling showed overall lower binding energy values of WA for cysteines compared with the previously identified GPX4-targeting agents altretamine and ML162 (Supplemental Table 4). The conserved Cys107 is predicted to be the most favorable site for WA-mediated alkylation (Figure $1 \mathrm{H}$ and Supplemental Video 3). Taken together, these data suggest a dual action mechanism of WA-induced ferroptosis. At high dose, WA acts as a class II (GPX4-targeting) ferroptosis inducer, while at cytotoxic medium dose, ferroptosis is induced through an alternative mechanism.

HMOX1-mediated increase in the labile Fe(II) pool boosts ferroptosis. To explore the mechanism of action of ferroptosis induction in response to a medium dose of WA, we performed a genomewide transcriptome analysis in IMR-32 and SK-N-SH cells. Interestingly, we identified heme oxygenase-1 (HMOX1) as the top upregulated gene by WA in both IMR-32 and SK-N-SH neuroblastoma cells. Ingenuity Pathway Analysis (IPA) of the data set indicated nuclear factor-like 2-mediated (NRF2-mediated) oxidative stress as one of the top signaling pathways induced by WA, but not WN, in IMR-32 cells (Figure 2, A and B) and SKN-SH cells (Supplemental Figure 2, A and B). This observation of a similar transcriptome profile in both subtypes is remarkable, given that there can be large disparities in the way that neuroblastoma subtypes respond to drug treatments. Validation of WAmediated regulation of HMOX1 confirmed its upregulation at the mRNA (Figure 2C) and the protein level (Figure 2D) in response to medium dose of WA, while this was not the case in response to a noncytotoxic low dose or a cytotoxic high dose of WA. HMOX1 expression is controlled by the NRF2/antioxidant response element signaling pathway (23). The NRF2 transcription factor is kept in check by Kelch-like ECH-associated protein 1-dependent (KEAP1-dependent) degradative ubiquitination (23). In line with this, we observed decreased levels of KEAP1 along with increased levels of NRF2 in conditions in which HMOX1 is upregulated (Figure 2D). Pull-down of WA from lysates of cells treated with biotinylated WA revealed KEAP1 binding in the presence of a medium dose of WA (Figure 2E). Thus, while a medium dose of WA did not bind to GPX4, it bound KEAP1, resulting in strong upregulation of HMOX1. We found that pharmacological inhibition of HMOX1 activity strongly inhibited ferroptotic cell death after exposure to a medium dose of WA, while a transient delay was observed upon exposure to a high dose (Figure 2F). Similarly, RNAimediated knockdown of HMOX1 suppressed ferroptosis in response to a medium dose of WA, while no protection was observed upon exposure to a high dose of WA (Figure 2, G and $\mathrm{H})$. Both findings indicate a cytotoxic instead of protective role of HMOX1 in ferroptosis induced by a medium dose of WA. This seems counterintuitive because HMOX1 activity is part of an antioxidant and detoxifying system.

HMOX1 detoxifies heme into biliverdin, releasing carbon monoxide and Fe(II) (24). Addition of the HMOX1 substrate hemin sensitized cells to WA-induced ferroptosis in response to a medium dose, but not a high dose (Figure 2I). Like cells treated with a medium dose of WA, we found that administration of hemin induces a strong upregulation of HMOX1 (Figure 2J) and its dose-dependent cytotoxicity is blocked by an HMOX1 inhibitor (ZnPP) or a panel of ferroptosis inhibitors (Figure 2K). Essentially, the detoxification of heme by HMOX1 results in the release of labile $\mathrm{Fe}$ (II) (24), which has been proposed to be involved in oxidative stress-mediated cell death (25). In line with our hypothesis, we revealed a time-dependent increase in labile $\mathrm{Fe}$ (II) in response to WA using an Fe(II)-selective probe, RhoNox-1 (26) (Figure 2L). Adding hemin to IMR-32 cells also increased WA-induced elevation of the labile Fe(II) pool (Figure 2M). In addition, increasing the $\mathrm{Fe}$ (II) pool in the cell (Supplemental Figure 2C) using ferrous ammonium sulfate $\left[\left(\mathrm{NH}_{4}\right)_{2} \mathrm{Fe}\left(\mathrm{SO}_{4}\right)_{2}\right]$ induced cell death that could be blocked by ferroptosis inhibitors (Figure $2 \mathrm{~N}$ ), suggesting that increasing the labile $\mathrm{Fe}$ (II) pool as such is sufficient to initiate ferroptosis in neuroblastoma cells. Note that, consistent with the observed upregulation of FTH1 in our transcriptome analysis (Figure 2B and Supplemental Figure 2B), we also observed a stronger upregulation of FTH1 in response to a medium dose of WA, compared with a high dose (Supplemental Figure 2D). This response may indicate an attempt to buffer the increase in Fe(II) that followed WA-induced excessive activation of HMOX1. In summary, ferroptosis induced by a medium dose of WA is dependent on an increase in the labile $\mathrm{Fe}$ (II) pool upon excessive activation of HMOX1. Moreover, hemin- or $\left(\mathrm{NH}_{4}\right)_{2} \mathrm{Fe}\left(\mathrm{SO}_{4}\right)_{2}$-induced increase in labile $\mathrm{Fe}(\mathrm{II})$ is sufficient to induce ferroptosis.

WA eradicates high-risk neuroblastoma tumors and shows lower relapse rates compared with etoposide. Because WA was effective in killing a panel of high-risk neuroblastoma cells in vitro, we next sought to investigate the potency of WA to suppress tumor 
A

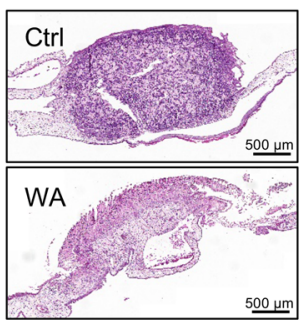

B

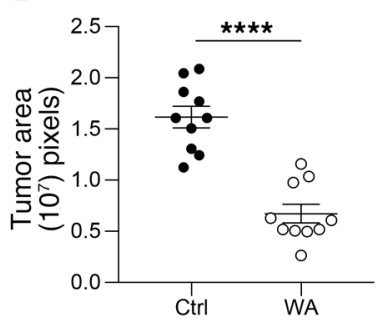

C

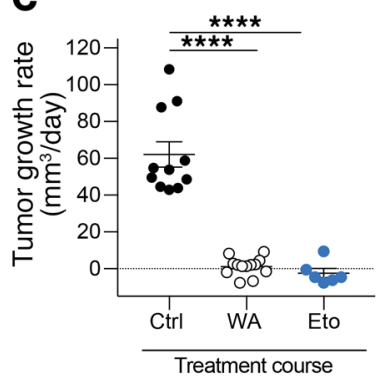

$\mathbf{F}$

E

Ki67
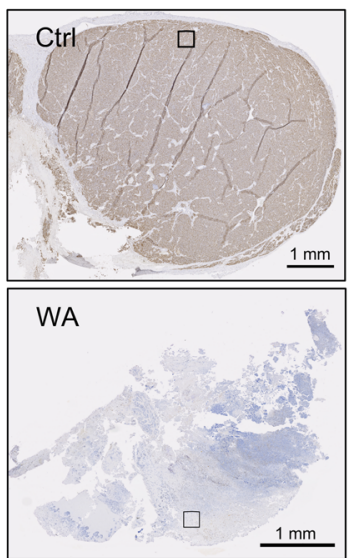

G

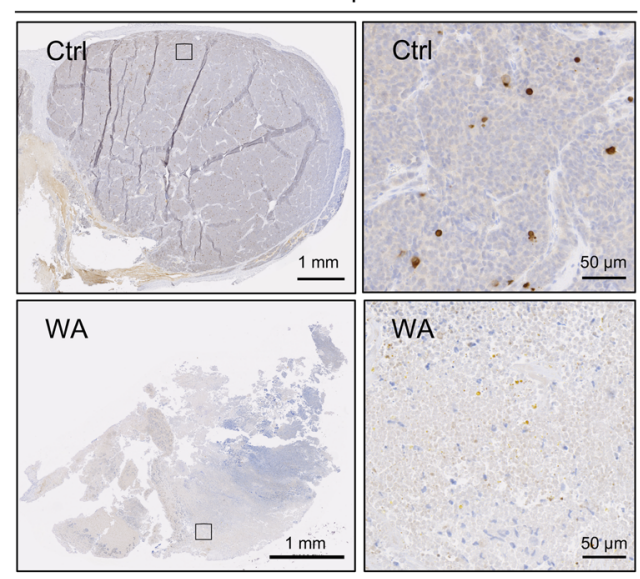

I

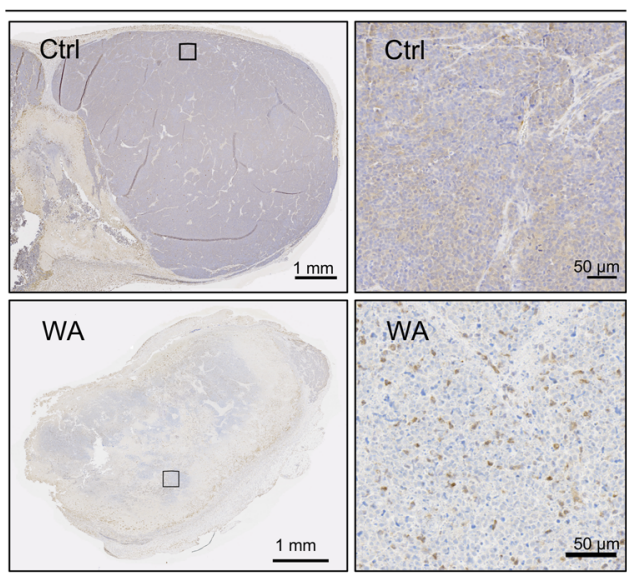

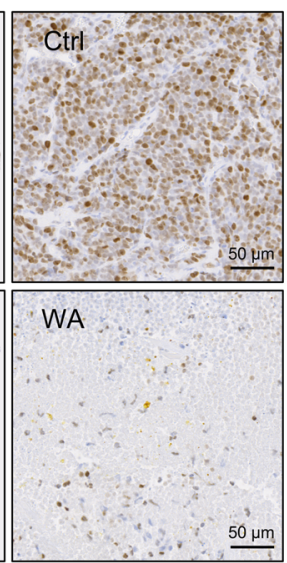
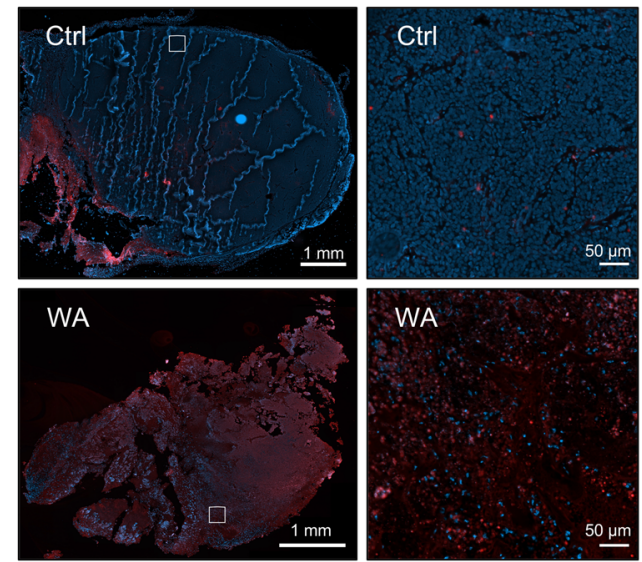

H

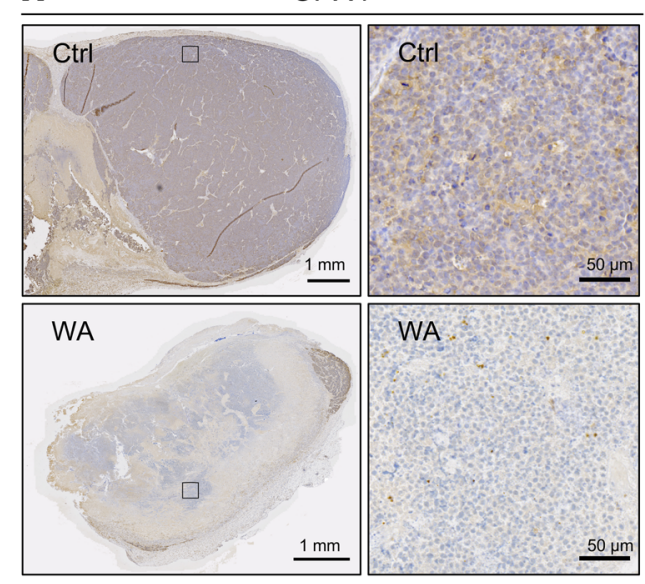

J

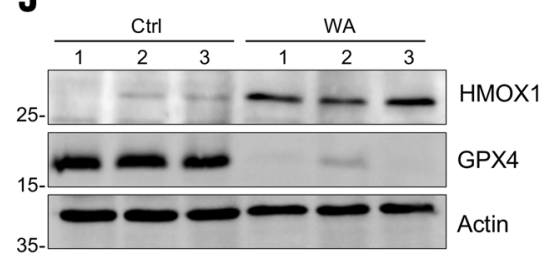

F TUNEL

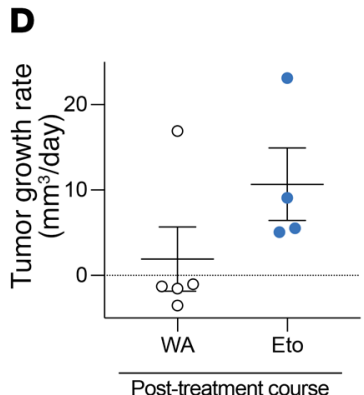

Figure 3. WA-eradicated highrisk neuroblastoma tumors show decreased GPX4 expression, increased HMOX1 expression, and decreased relapse rates. (A and B) Light microscopic pictures of $\mathrm{H} \& \mathrm{E}$ staining of tumor sections (A) and quantification of tumor size area (B) representing effect of WA and vehicle (Ctrl) on SH-EP-derived tumors in a chicken chorioallantoic membrane (CAM) model. The combined results of 2 independent experiments are shown. Error bars represent SEM; $n=10$ (Ctrl), $n=10$ (WA). (C) Quantification of tumor growth rates after therapeutic treatment regime $\left(100-200 \mathrm{~mm}^{3}\right.$ at the start of treatment) with vehicle (Ctrl), WA, or etoposide (Eto). The results of 1 experiment for etoposide and combined results of 2 independent experiments for WA are shown. Each point indicates an individual mouse. Error bars represent SEM; $n=11$ (Ctrl), $n=13$ (WA), $n=6$ (Eto). (D) Quantification of tumor growth rates in mice after termination of treatment with WA and Eto (tumor size cutoff below $180 \mathrm{~mm}^{3}$ at time of termination of the treatment). (E-I) Immunohistochemical staining revealing proliferation using Ki67 antibody (E), DNA fragmentation using TUNEL staining (F), caspase-3 activation using anti-active caspase-3 antibodies (C), GPX4 (H), and HMOX1 expression level (I) in tumor sections after therapeutic treatment regime with vehicle (Ctrl) or WA. (J) Western blot revealing GPX4 and HMOX 1 in lysates of tumor sections after therapeutic treatment regime with vehicle (Ctrl) or WA. ${ }^{* * * *} P<0.0001$, 2-tailed $t$ test (B), 1-way ANOVA (C). 
growth. Before exploring xenograft in vivo models, we investigated the effect of WA on an ex vivo tumor growth model using a chicken chorioallantoic membrane assay. These tumors were treated for 3 consecutive days with WA or vehicle (Ctrl). Analysis of the histological sections demonstrated eradication of the tumor after WA treatment (Figure 3A). Quantification of the tumor size revealed a significant reduction after WA inoculation (Figure 3B). Next, to test the chemopreventive potential of WA in an experimental neuroblastoma xenograft mouse model, we subjected subcutaneously growing IMR-32 cells to WA or vehicle (Ctrl) starting 1 day after implantation. Daily monitoring of the tumor size demonstrated a significant decrease in tumor growth rate after WA treatment regime (Supplemental Figure 3, A and B). To validate the chemotherapeutic potential of WA, we first started the intratumoral WA treatment when the tumor reached a volume of approximately $60 \mathrm{~mm}^{3}$. This approach resulted in a significant retardation in tumor growth (Supplemental Figure 3, C and D). Next, we compared the efficacy of intratumoral WA treatment with etoposide in vascularized neuroblastoma tumors of approximately 100-200 $\mathrm{mm}^{3}$. WA was as effective as the standard chemotherapeutic etoposide in suppressing the tumor growth rate (Figure 3C and Supplemental Figure 3E). Upon termination of the treatment, we noted that 4 out of 5 mice did not show any relapse, while all mice treated with etoposide showed a relapse within 10-15 days (Figure 3D and Supplemental Figure $3 \mathrm{~F}$ ). This could be of utmost importance considering that as many as $50 \%-60 \%$ of children with high-risk neuroblastoma will eventually die upon relapse.

Tumor regression occurs through lipid peroxidation-mediated cell death. Analysis of the tumor samples allowed us to verify whether the mode of action of the cytotoxicity of WA was similar in vivo compared with our experiments performed in neuroblastoma cells. Immunohistochemical staining of tumor sections with a marker for proliferation (anti-Ki67) showed a nearly absent staining in WA-treated tumors compared with tumors treated with vehicle (Ctrl) (Figure 3E). In addition to tumor growth inhibition, we observed that WA induced massive cell death reflected by TUNEL positivity (Figure 3F) without caspase-3 activation (Figure $3 \mathrm{G})$. The cell death induced by WA in the tumor is associated with an infiltration of large numbers of CD45-positive immune cells (Supplemental Figure 3G). Moreover, in accordance with our proposed mechanism of action, WA-treated tumors showed a drop in GPX4 expression (Figure 3, H and J) and a concomitant increase in HMOX1 expression levels (Figure 3, I and J). Because lipid peroxidation is a key feature in ferroptosis, we finally performed liquid chromatography-mass spectrometry-based redox-lipidomics analysis (27) of tumors treated with WA or vehicle (Ctrl) (Figure 4 and Supplemental Figure 4). This analysis revealed an accumulation of peroxidized lipid species in WA-treated tumors reflected by a significant increase in the contents of oxygenated polyunsaturated species in several classes of phospholipids. This effect was particularly robust in phosphatidylglycerol (PG) and phosphatidylinositol (PI) but also observable in other major classes of phospholipids, such as phosphatidylcholine (PC), phosphatidylethanolamine (PE), and phosphatidylserine (PS), with the notable exception of cardiolipin (CL). The elevated levels of oxygenated (ox) species of PG and PI contained hydroperoxy- (+2O) or dihy- droperoxy- $(+4 \mathrm{O})$ groups, which is likely due to the insufficiency of GPX4-driven mechanism of reduction of phospholipid hydroperoxides to respective hydroxy-derivatives (Figure 4A). Oxidation products in other classes of phospholipids were represented by mono-oxygenated species (Figure 4B), likely hydroxy-derivatives that are usually formed as products of GPX4-dependent reductive metabolism. In addition, significant elevation of the contents of several types of lysophospholipids was found in WA-treated tumors. The high levels of lysophospholipids were detected in PC, PE, PS, and CL as LPC, LPE, LPS, and monolyso-cardiolipin (MLCL), respectively (Figure 4C). It is interesting that no elevated levels of lysophospholipids were found for PG and PI, whose oxidation yielded hydroperoxy-derivatives (Figure 4A). Despite the accumulation of MLCL in WA-treated tumors, we did not observe an accumulation of any oxygenated CL species (Supplemental Figure 4). Given that mono-oxygenated CL species are highly specific for the execution of intrinsic apoptotic program (28), these data corroborate that WA induced a caspaseindependent form of cell death in neuroblastoma tumors with clear features of lipid peroxidation compatible with the induction of ferroptosis. Together, these data indicate the potential of WA to induce massive cell death in neuroblastoma tumors with clear features of ferroptosis, which subsequently triggers the infiltration of high numbers of immune cells.

Nano-targeting of WA avoids systemic side effects and suppresses tumor growth. Because of poor solubility of WA in water and severe weight loss-related adverse effects upon systemic injection (Figure 5D), we formulated WA in a novel amphiphilic degradable $\mathrm{pH}$ sensitive nanocarrier to allow systemic administration in a neuroblastoma xenograft model (Figure $5 \mathrm{~A}$ ). In vitro characterization of the WA nanoparticles (WA-NPs) revealed a size of approximately 10-50 nm (Figure 5B) and an equal cytotoxic efficacy in IMR-32 cells compared with nonencapsulated WA (Figure 5C). Note that we tested 2 other formulation approaches, which were unsuccessful. Using a different polymeric excipient for physical encapsulation requiring tetrahydrofuran for cosolubilization of polymer and drug, followed by solvent displacement in aqueous medium (29), we observed cytotoxicity of the empty vehicle (Empty-NP2) (Supplemental Figure 5A). Commonly used polymer amphiphilic excipients for drug encapsulation such as those based on poly (ethyleneglycol-block-lactic acid) and poly(ethyleneglycol-block[glycolic-co-lactic acid]) were not capable of stably accommodating WA because of precipitation of WA crystals in aqueous medium. Using a WA-polymer conjugation approach by covalent linking of a hydrophilic polymer [poly( $N, N$-dimethylacrylamide)] to a hydroxyl moiety of WA via an ester bond (30), we observed minor cytotoxic activity of WA-NP3 in IMR-32 neuroblastoma cells (Supplemental Figure 5A), and no tumor regressing activity in vivo (Supplemental Figure 5B). To confirm enhanced targeting to the tumor site due to enhanced permeability and retention (EPR) effect (31), nanoparticles fluorescently labeled with Cy5 (Cy5-NPs) were injected intraperitoneally (Figure 5E). Systemic application of WA-NPs to mice bearing tumors with a volume of approximately $60-70 \mathrm{~mm}^{3}$ significantly suppressed the growth of neuroblastoma tumors (Figure $5 F$ ) without significant weight loss (Figure 5G). In conclusion, WA seems to be a promising candidate for further therapeutic exploitation using novel targeting strategies such as cancer nanomedicine. 

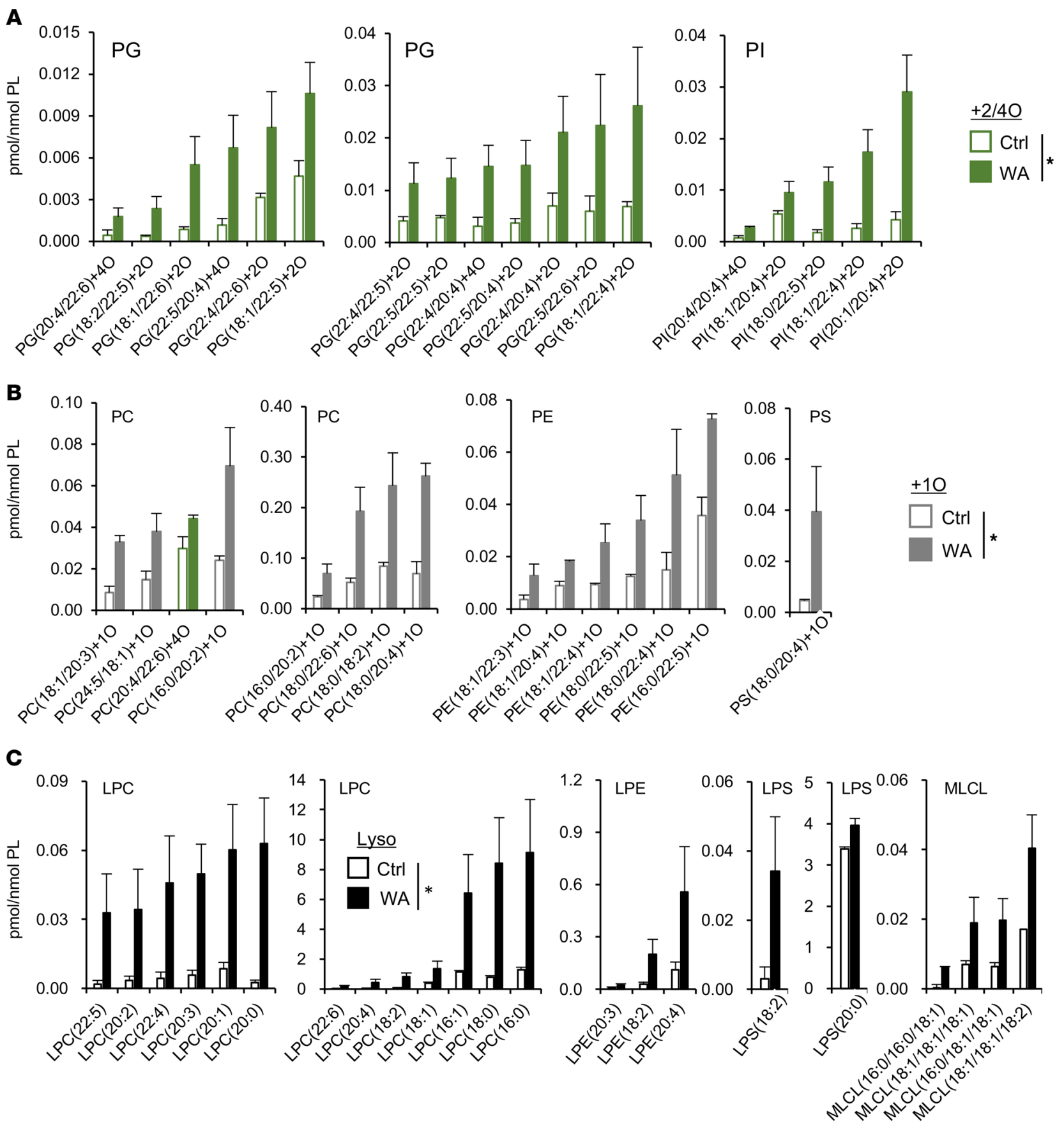

Figure 4. Oxidative lipidomics profiles show massive lipid peroxidation in neuroblastoma tumors treated with WA. (A and B) Levels of oxygenated phospholipid species, with significantly higher levels (33 species) detected in neuroblastoma tumors from mice treated with WA compared with vehicletreated mice (Ctrl). Error bars represent SEM; $n=3$. (C) Levels of lysophospholipids, with significant increase (22 species) in neuroblastoma tumors treated with WA. Error bars represent SEM; $n=3$. PL, phospholipids; PG, phosphatidylglycerol; PI, phosphatidylinositol; PC, phosphatidylcholine; PE, phosphatidylethanolamine; PS, phosphatidylserine; L, lyso; MLCL, monolyso-cardiolipin. ${ }^{*} P<0.05$, 2-tailed $t$ test $(\mathbf{A}-\mathbf{C})$.

\section{Discussion}

In the present study, the natural phytochemical WA isolated from Withania somnifera roots (17) was identified as a novel natural compound that kills a panel of high-risk neuroblastoma cells (mostly quite etoposide and cisplatin resistant) through a novel double-edged ferroptosis-inducing mechanism (Figure 5H). On one hand, WA acts as a class II FIN (6), which resulted in a rapid drop in expression and activity of GPX4. GPX4 protects mem- branes against lipid peroxidation, and its targeted degradation typically results in ferroptosis (32). On the other hand, a medium dose of WA induced a massive upregulation of HMOX1, which was followed by an increase in cellular labile $\mathrm{Fe}(\mathrm{II})$, and consequent ferroptosis. The mechanism by which WA upregulates HMOX1 occurs via targeting of KEAP1, the negative regulator of NRF2, as we recently reported for endothelial cells (15). Pharmacological inhibition or knockdown of HMOX1 blocked ferroptosis 
A
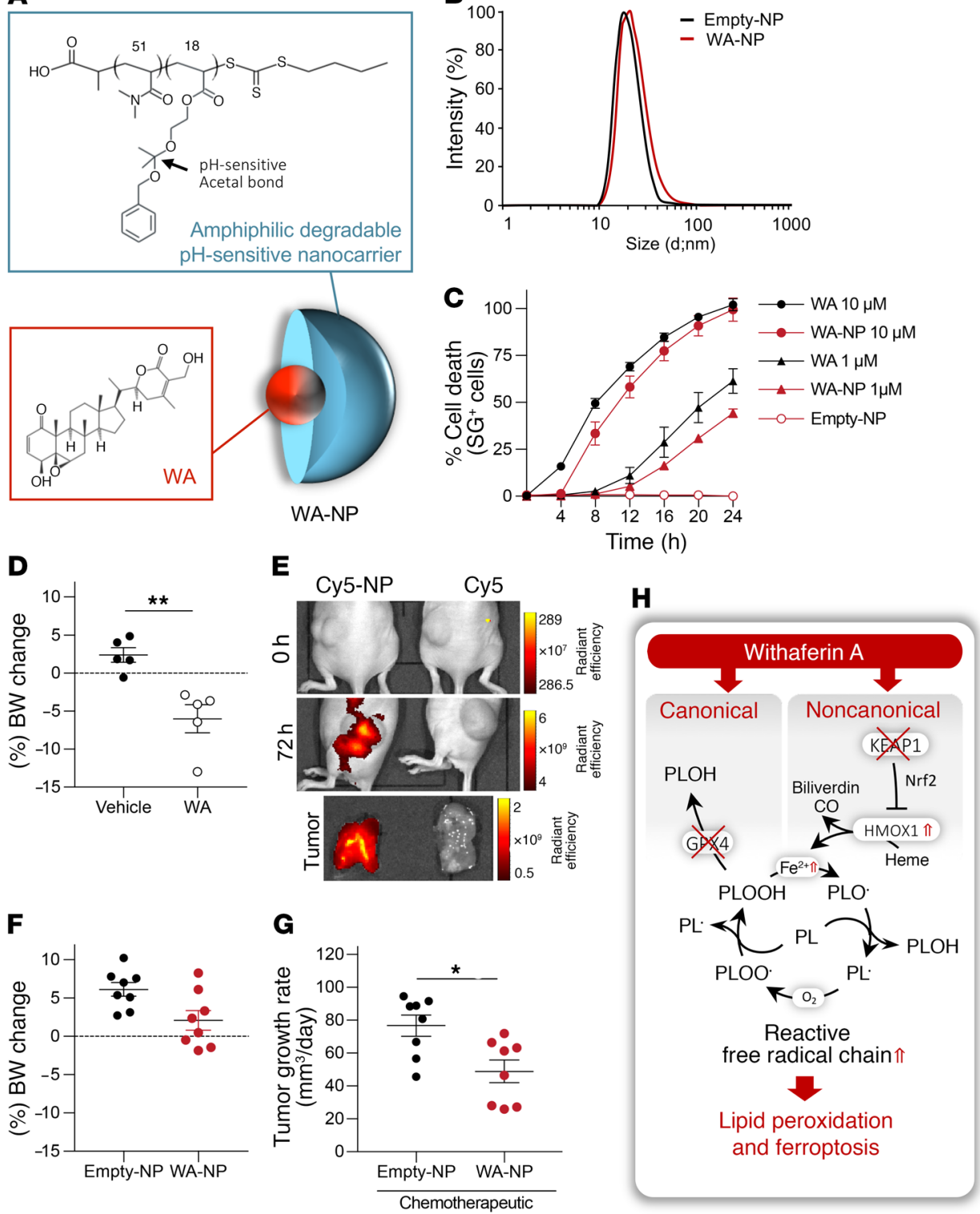

Figure 5. Nano-targeting of WA avoids systemic side effects and suppresses tumor growth. (A) Schematic representation of the amphiphilic degradable $\mathrm{pH}$-sensitive nanoparticle encapsulating WA (WA-NP). (B) Histogram indicating the size of empty (Empty-NP) and WA-encapsulated nanoparticles (WA-NP). d;nm means diameter of nanoparticles in nanometer. (C) Percentage of cell death in function of time triggered by WA-NPs, Empty-NPs, and WA in IMR-32 cells. (D) Percentage of body weight change in mice 4 days after daily i.p. injection of WA and vehicle. (E) In vivo fluorescence images of neuroblastoma tumor xenografts in nude mice injected i.p. with Cy5 and Cy5-loaded nanoparticles (Cy5-NPs). (F) Percentage of body weight change in mice 9 days after daily i.p. injection of empty (Empty-NP) or WA-encapsulated nanoparticles (WA-NP). (C) Quantification of tumor growth rates after therapeutic treatment regime (60-70 $\mathrm{mm}^{3}$ at the start of injection) with empty (Empty-NP) or WA-encapsulated nanoparticles (WA-NP). Each point indicates an individual mouse. Error bars represents SEM; $n=8$ per group. (H) PL $\bullet$, phospholipid radical; PLOO•, phospholipid peroxyradical; $\mathrm{LOOH}$, phospholipid hydroperoxide; PLOH, phospholipid alcohol. Schematic representation of a double-edged ferroptosis induction mechanism upon WA exposure. WA induces uncontrolled lipid peroxidation and ferroptosis through inactivation of GPX4 (canonical ferroptosis induction) and excessive activation of HMOX1 followed by an increase in the labile $\mathrm{Fe}$ (II) pool (noncanonical ferroptosis induction). Inhibiting the lipid detoxification process, by inactivating GPX4, as well as fueling the reactive free radical chain process by increasing the labile $\mathrm{Fe}(\mathrm{II})$ pool effectively boosts ferroptosis. ${ }^{*} P<0.05 ;{ }^{*} P<0.001$, 2 -tailed $t$ test ( $\mathbf{D}$ and $\mathbf{G}$ ). induced by a medium dose of WA, which indicates that elevated HMOX1 expression and activity have a direct role in ferroptotic cell death induced by WA.

Recently, 2 studies reported opposite roles for HMOX1 in erastin-induced ferroptosis. A protective role was proposed in hepatocellular carcinoma (33) and glioblastoma (34), while a cytotoxic role was observed in fibrosarcoma cells (35). We propose that the level of HMOX1 upregulation might determine whether HMOX1 promotes or inhibits ferroptosis. This biological phenomenon resembles hormesis: a moderate level of HMOX1 is cytoprotective, while excessive HMOX1 activation becomes cytotoxic because of an increase in labile Fe(II) due to insufficient buffering capacity by ferritin $(25,36)$. This mechanism of action is underscored by the observation that the increase in HMOX1 expression in response to a medium dose of WA was 10-fold higher than the increase in FTH1 expression. In addition, we identified that increasing the labile $\mathrm{Fe}(\mathrm{II})$ pool by adding $\left(\mathrm{NH}_{4}\right)_{2} \mathrm{Fe}\left(\mathrm{SO}_{4}\right)_{2}$ or hemin is sufficient to trigger ferroptosis in neuroblastoma cells.
Additional research is needed to verify whether this noncanonical ferroptosis induction by excessive activation of HMOX1 and increase in cellular labile Fe(II) could create new opportunities to effectively kill other types of tumor cells.

Neuroblastoma patients have increased levels of iron and HMOX1 expression in the tumor tissue and increased ferritin levels in plasma $(37,38)$. Therefore, iron chelation strategies (11, 39) or HMOX1 inhibition are currently explored to reduce tumor growth (38). Our data, however, propose an opposite strategy. In the present study, we show that both high levels of labile Fe(II) and excessive activation of HMOX1 are important to eradicate neuroblastoma through ferroptotic action mechanisms. Therefore, the high level of iron, GPX4, and HMOX1 expression in cancer cells can be an Achilles' heel, as it makes tumor cells vulnerable to ferroptosis induction upon therapeutic targeting $(11,40)$.

It is remarkable that the level of protection by Fer1 against hydroperoxide formation and ferroptosis in response to RSL3 and a high dose of WA (class II FINs) was incomplete compared with the 
level of protection in response to erastin (a class I FIN). This might be specific to neuroblastoma cells, although a similar incomplete protection against hydroperoxides by Fer1 was previously observed in Pfa cells upon GPX4 depletion (19) and in hydrogen peroxidetreated mouse embryonic fibroblasts (41) and HT1080 cells (9). Alternatively, WA might have additional targets that promote iron-dependent cell death independent of Fer1-sensitive ferroptosis, similar to other covalent modifying agents such as phenethyl isothiocyanate $(9,42)$ and piperlongumine $(43)$. Notably, Fer1 does block ferroptosis completely in $\mathrm{H}_{2} \mathrm{O}_{2}$-hypersensitive cells in which the selenocysteine in GPX4 is mutated to cysteine (41). This indicates that $\mathrm{H}_{2} \mathrm{O}_{2}$ induces ferroptosis that is Fer1-inhibitable. However, in WT cells, you need to overcome GPX4-mediated protection against hydroperoxide formation by using a very high concentration of $\mathrm{H}_{2} \mathrm{O}_{2}$ (millimolar range). Considering the mechanism of action of Fer1 as a free radical trap (44), we argue that Fer1 is incapable of protecting against excessive levels of lipid ROS induced by, for instance, $\mathrm{H}_{2} \mathrm{O}_{2}$ or WA, although both induce ferroptosis.

The recent identification of the FDA-approved anticancer agents altretamine (45), sorafenib (46), and silica nanoparticles (47) as ferroptosis inducers creates high expectations of newly identified ferroptosis-inducing agents (32). Our study adds WA as the first ferroptosis-inducing natural compound that targets GPX4 (class II FIN), which may function as an interesting lead anticancer agent. Although limiting cysteine uptake and GSH synthesis has been reported as a mechanism to induce ferroptosis, this may be insufficient to kill certain cancer cells. In several types of cancers, upregulation of the SLC7A11 protein, a subunit of system $\mathrm{X}_{\mathrm{C}}^{-}$, has been observed. This overcomes ferroptosis triggered by system $\mathrm{X}_{\mathrm{C}}{ }^{-}$inhibitors $(48,49)$. Cysteine can also be supplied via other transporters, such as the system ASC transporters (50). In addition, the induction of GSH depletion in some cancer cells can be compensated by other thiol-containing antioxidants such as thioredoxin enzymes (51). Therefore, direct targeting and inactivation of GPX 4 by class II FINs such as WA might circumvent adaptive mechanisms of cancer cells that bypass inhibition of system $\mathrm{X}_{\mathrm{C}}{ }^{-}$or GSH depletion.

High-risk neuroblastoma patients have poor prognosis and show large disparities in drug response due to genetic instability and intratumoral heterogeneity (1). We show that WA is equally effective in killing neuroblastoma cells with or without MYCN amplification, indicating that WA has broad therapeutic potential to kill high-risk neuroblastoma independent of its heterogeneity. It is remarkable that WA-treated mice that showed a strong regression of the tumor showed no signs of relapse upon termination of the treatment, in contrast to etoposide. This is likely due to the resistance of high-risk neuroblastoma cells to etoposide, which might allow regrowth of the tumor upon termination of the treatment. Etoposide-induced tumor regression likely acts mainly by targeting angiogenesis (52), and WA by targeting both the vasculature (53) and the neuroblastoma tumor.

It is a central theme in cancer therapy that polymeric implants (54) or targeted delivery through nanoparticles $(40,47)$ could increase efficacy of cancer treatments and bypass resistance pathways. To increase the targeting of WA to the tumor, we generated WA-encapsulated nanoparticles using a novel amphiphilic degradable $\mathrm{pH}$-sensitive nanocarrier. This nanomedicine approach allowed systemic application and suppressed tumor growth.
This implies that WA might be a promising candidate for further therapeutic exploitation.

In summary, we have shown the high effectiveness of WA in eradicating high-risk neuroblastoma due to a double-edged ferroptosis mechanism. A high dose of WA makes tumor cells vulnerable to ferroptosis through GPX4 targeting and inactivation leading to canonical ferroptosis induction, while a medium dose of WA activates the NRF2 pathway through KEAP1 targeting. This noncanonical induction of ferroptosis is characterized by an increase in intracellular labile Fe(II) upon excessive activation of HMOX1, which is sufficient to induce ferroptosis. It will be interesting to screen for other compounds that upregulate HMOX1 or the labile $\mathrm{Fe}$ (II) pool. Additional research to explore how sensitizing therapies (by, e.g., hemin cotreatment), use of more potent WA analogs (55), or targeting strategies can further improve the effectiveness of WA will expand the therapeutic potential of WA in malignancies such as high-risk neuroblastoma.

\section{Methods}

Antibodies and reagents. The following antibodies were used in this study: cleaved caspase-3 (Cell Signaling, 9661), $\beta$-tubulin (Abcam, ab21058), actin (MP Biomedicals, 69100), GPX4 (Abcam, ab41787), CD45 (BD Biosciences, 550539), Ki67 (Cell Signaling, 12202S), HMOX1 (Enzo Life Sciences, ADI-SPA-896-F), NRF2 (Abcam, ab62352), and KEAP1 (Proteintech, 10503-2-AP). The following reagents were used: BODIPY 581/591 C11 probe (Invitrogen, D-3861), DHR123 probe (Thermo Fisher Scientific, D23806), SytoxGreen (Thermo Fisher Scientific, S7020), streptavidin-Sepharose beads (GE Healthcare, 17-5113-01), diaminobutyric acid (DAKO, K3468), antigen retrieval solution (DAKO, s2031), erastin (Sigma-Aldrich, E-7781; Selleckchem, S7242), withaferin A (Altavista Phytochemicals), FeRhoNox-1 (Goryo Chemical, GC901), FAS (Sigma-Aldrich, F7002), hemin (Sigma-Aldrich, H9039), ML162 (Aobious, AOB1514), RSL3 (Selleckchem, S8155), and Matrigel matrix (Corning, 356231). DEVD-AMC (Pepta Nova, 3171-V) was used at 20 $\mu \mathrm{M}$. Nec1 (Calbiochem, 480065) was used at $10 \mu \mathrm{M}$. Fer1 (Xcess Biosciences, 053224) was used at $500 \mathrm{nM}$. DFO (Sigma-Aldrich, D-9533) was used at $100 \mu \mathrm{M}$ and $1 \mathrm{mM}$. Necrosulfonamide (NSA; Calbiochem, 480073) was used at $2.5 \mu \mathrm{M}$. BHA (Sigma-Aldrich, B-1253) was used at $100 \mu \mathrm{M}$. DecylQ (Sigma-Aldrich, D7911) was used at $10 \mu \mathrm{M}$. Nordihydroguaiaretic acid (NDGA; Sigma-Aldrich, N-5023) was used at $10 \mu \mathrm{M}$. $\alpha$-Tocopherol (Sigma-Aldrich, T3251) was used at $100 \mu \mathrm{M}$. Ciclopirox olamine (CPX; Sigma-Aldrich, C0415) was used at $5 \mu \mathrm{M}$ and $10 \mu$ M. FLT3 inhibitor III (Merck, 343022) was used at 500 nM. U0126 (Sigma-Aldrich, U120) was used at $10 \mu \mathrm{M}$. The caspase peptide inhibitor Z-VAD-FMK (Bachem, N-1510) was used at $10 \mu \mathrm{M}$. The heme oxygenase-1 inhibitor (Enzo Life Sciences, ALX-430-049-M025) was used at $1.2 \mu \mathrm{M}$. Synthesis of biotinylated WA has been described previously and was provided by P. Vanderveken (15).

Cell lines. IMR-32, SK-N-SH, Kelly, NB69, and CHP-134 cells were cultured in RPMI 1640 medium supplemented with 10\% FCS and L-glutamine (1 mM). NLF cells were cultured in RPMI 1640 medium supplemented with $10 \%$ FCS, L-glutamine $(1 \mathrm{mM})$, and sodium pyruvate $(1 \mathrm{mM})$. SH-EP cells were cultured in DMEM supplemented with $10 \%$ FCS, L-glutamine $(1 \mathrm{mM})$, and sodium pyruvate $(1 \mathrm{mM})$. SH-SY5Y, SK-N-AS, SK-N-BE(2)C, and SK-N-DZ cells were cultured in DMEM supplemented with $10 \%$ FCS and L-glutamine (1 mM). IMR-32 and SK-N-SH cells were obtained from Jo Vandesompele, Ghent University 
Hospital, Medical Research Building, Ghent, Belgium. CHP-212 and NB69 cells were obtained from Simone Fulda, Institute for Experimental Cancer Research in Pediatrics, Goethe-University. Other neuroblastoma cells were obtained from Paul G. Ekert, Cancer Research, Murdoch Children's Research Institute, Royal Children's Hospital.

In vivo mouse studies. Briefly, IMR-32 cells were collected and suspended at a density of $8 \times 10^{6}$ in $70 \mu \mathrm{l}$ PBS and then mixed with 70 $\mu \mathrm{l}$ of Matrigel matrix. The cell suspension was injected into BALB/c nude mice subcutaneously. In the prevention study, the next day mice were randomized to 2 groups, and $200 \mu$ of vehicle (PBS 50\%, DMSO 20\%, PEG300 30\%) or WA (vehicle containing $4 \mathrm{mg} / \mathrm{kg}$ of WA) was injected subcutaneously to the same site for 4 consecutive days. Tumor size was measured for 2 weeks. In the therapeutic study, a setup of an interrupted bisequential or continuous intratumoral WA treatment regime was used when the tumor reached an average volume of either 60 or $100-200 \mathrm{~mm}^{3}$, respectively. In the bisequential treatment, 4 consecutive days of WA ( $4 \mathrm{mg} / \mathrm{kg}$ ) or vehicle, then 6 days of recovery was followed by 3 more daily treatments. In a continuous regime, WA $(4 \mathrm{mg} / \mathrm{kg})$ was injected daily for maximum 20 days. In the therapeutic study with WA-NPs, when the tumors reached a volume of $60 \mathrm{~mm}^{3}$ on average, mice were injected intraperitoneally and then twice peritumorally (days 1 and 5) with WA-NPs or Empty-NPs for a period of 2 weeks. To verify effective tumor targeting, mice injected intraperitoneally with Cy5-containing nanoparticles or nonencapsulated Cy5 for 3 consecutive days were imaged using the IVIS Spectrum In Vivo Imaging System (PerkinElmer). Tumors were measured by electronic caliper every day in 3 dimensions (length, width, height) with the formula $V=1 / 2 \times W \times H \times L$, where $V$ is the tumor volume, $L$ is the length, $W$ is the width, and $H$ is the height. For all local injections mice were anesthetized by isoflurane.

Chicken chorioallantoic membrane assay. Chorioallantoic membrane (CAM) assay was performed as previously described (56). Briefly, $1 \times$ $10^{6} \mathrm{SH}$-EP cells were resuspended in $10 \mu \mathrm{l} \mathrm{NaCl} 0.9 \%$ and $10 \mu \mathrm{l}$ Matrigel matrix implanted on the CAM of fertilized chicken eggs on day 8 of incubation and allowed to form tumor. The next day, tumor was treated with WA $(2.5 \mu \mathrm{M})$ or DMSO daily for 3 days. Four days after inoculation, tumors were excised with the surrounding CAM, fixed in $4 \%$ paraformaldehyde, embedded in paraffin, cut in $5-\mu \mathrm{m}$ sections, and stained with 1:1 hematoxylin and $0.5 \%$ eosin solution for histological analysis. Images were taken with a 3DHISTECH MIDI Confocal scanner PlanApo $\times 20 / 0.8$ NA dry objective and a Confocal PCO.edge camera, and the tumor area was analyzed using Image J software (NIH).

Lentiviral transduction. GPX4 overexpression in IMR-32 cells was achieved by lentiviral transduction. Briefly, $1 \times 10^{6} \mathrm{HEK} 293 \mathrm{~T}$ cells were transfected using calcium phosphate with lentiviral expression vector p442-PL1 containing murine active GPX4 or inactive GPX4 (Sec/Ser) in combination with lentiviral packaging vectors pMD2-VSVG and pCMV-DR8.91. The medium was changed after 6 hours, and virus was collected 48 hours after transfection. The virus-containing supernatant was then used to transduce IMR-32 cells. The GPX4-overexpressing cells were then sorted and collected based on expression of GFP fluorescence by use of flow cytometry. Lentiviral expression vector p442PL1 was obtained from Marcus Conrad, Helmholtz Zentrum München, Institute of Developmental Genetics.

Analysis of cell death and caspase-3 activity. Cell death and caspase activity were measured as previously described using the FLUOstar Omega fluorescence plate reader (BMG Labtech GmbH) (57). Brief- ly, cells were seeded in a 96-well plate, and all experiments were performed in triplicate. The next day cells were preincubated with the desired inhibitors for 1 hour or 4 hours (for DFO) then treated with stimuli at desired concentrations in the presence of SytoxGreen $(1.7 \mu \mathrm{M})$ and DEVD-AMC $(20 \mu \mathrm{M})$. Afterward, the plate was transferred to a temperature- and $\mathrm{CO}_{2}$-controlled FLUOstar Omega fluorescence plate reader. The fluorescence intensity of SytoxGreen and DEVD-AMC was measured in function of the time at intervals of 1 hour with excitation/emission filters of 485/520 nm for SytoxGreen and 360/460 nm for DEVD-AMC. In each experiment, Triton $\mathrm{X}-100(0.05 \%)$ was used to induce lysis of the cells in at least 3 wells of the plate, and its signal intensity was used as $100 \%$ cell death reference. The percentage of the cell death was calculated by the formula (avg. SytoxGreen [stimuli] - avg. SytoxGreen [background]) / (avg. SytoxGreen [Triton X-100] - avg. SytoxGreen [background]) $\times$ 100. Caspase-3 activity was calculated: (avg. DEVD-AMC [stimuli] - avg. DEVD-AMC [background]) / (avg. DEVD-AMC [Triton $\mathrm{X}-100$ ] - avg. DEVD-AMC [background]) $\times 100$. The graphs were plotted in GraphPad Prism 6.

ROS and lipid ROS assay. Cellular lipid ROS were measured as previously described (9). Briefly, 600,000 cells per well were seeded in a 6-well plate. The next day, cells were treated with stimuli and harvested. Then cells were resuspended in $500 \mu \mathrm{l}$ PBS containing DHR123 $(1 \mu \mathrm{M})$ and C11-BODIPY (581/591) $(2 \mu \mathrm{M})$ and incubated for 10 minutes at $37^{\circ} \mathrm{C}$. Cells were then resuspended in $500 \mu \mathrm{l}$ of fresh PBS containing SytoxBlue $(1.25 \mu \mathrm{M})$ and analyzed by flow cytometry (BD FACSVerse, BD Biosciences).

GSH level measurement. Glutathione levels were measured using QuantiChrom Glutathione Assay Kit (BioAssay Systems, DIGT-250). Briefly, cells were seeded at $2 \times 10^{6}$ cells in a $5-\mathrm{cm}$ dish. The next day, cells were treated with DMSO or WA and erastin. Cells were collected and transferred to a new tube, and centrifuged at $425 \mathrm{~g}$ at $4^{\circ} \mathrm{C}$ for 5 minutes. The cell pellet was resuspended in $300 \mu \mathrm{M}$ of PBS and was lysed by sonication. The lysate was centrifuged at $18,500 \mathrm{~g}$ at $4^{\circ} \mathrm{C}$ for 10 minutes, and cleared lysate was used to determine the amount of GSH in the sample according to the instructions of the kit.

GPX 4 assay. Cells were seeded at $6 \times 10^{6}$ cells in $10-\mathrm{cm}$ dishes. The next day, cells were treated with WA or DMSO at the indicated time points. Cell were washed once with cold PBS and then collected and centrifuged at $425 \mathrm{~g}$ for 5 minutes. Then the cell pellet was resuspended in $200 \mu \mathrm{l}$ of lysis buffer $\left(\mathrm{KH}_{2} \mathrm{PO}_{4} / \mathrm{K}_{2} \mathrm{HPO}_{4}, \mathrm{pH} 7.4\right.$, containing EDTA 1 $\mathrm{mM}, \mathrm{KCl} 150 \mathrm{mM}$, CHAPS 0.1\%, $\beta$-mercaptoethanol $2 \mathrm{mM}$, protease inhibitor). Lysates were then centrifuged at 20, $800 \mathrm{~g}$ for 10 minutes at $4^{\circ} \mathrm{C}$. One hundred microliters of the lysate was added to $100 \mu \mathrm{l}$ of assay buffer $\left(100 \mathrm{mM} \mathrm{KH} \mathrm{PO}_{4} / \mathrm{K}_{2} \mathrm{HPO}_{4}, \mathrm{pH}\right.$ 7.4, containing EDTA $1 \mathrm{mM}$, peroxide-free Triton X-100 $0.1 \%$, GSH 4 mM, NADPH 0.4 $\mathrm{mM}$, and glutathione reductase $4 \mathrm{U} / \mathrm{ml}$ ) and transferred to a 96-well plate. Finally, $10 \mu \mathrm{l}$ of PCOOH $2.5 \mu \mathrm{M}$ substrate was added, and the amount of NADPH in the reaction mixture was determined kinetically by reading of absorbance value at $340 \mathrm{~nm}$ at 10 -second intervals over a period of 10 minutes. GPX activity was normalized to amount of protein in each sample and reported as percentage considering DMSO sample as $100 \%$ reference or nanomoles $\mathrm{PCOOH}$ reduced per minute per milligram of protein. $\mathrm{PCOOH}$ was obtained from Marcus Conrad, Helmholtz Zentrum München, Institute of Developmental Genetics.

Covalent docking of WA with GPX4. The human phospholipid hydroperoxide glutathione peroxidase 4 (GPX4) (PDB: 2OBI) (58) 
was retrieved from the Protein Data Bank (PDB) (https://www.rcsb. org) and prepared using the protein preparation wizard implemented in Schrödinger's Maestro suite (version 2015-3, Schrödinger LLC) by applying standard default settings and restrained minimization with OPLS 2005 force field (59). The small molecules altretamine (CID: 2123), withaferin A (CID: 265237), and ML162 (CID: 3689413) were downloaded from the PubChem database (https://pubchem.ncbi. nlm.nih.gov) and prepared using ligand preparation implemented in Schrödinger's Maestro (version 2015-3, Schrödinger LLC). Because of the good level of accuracy in determining bioactive conformations, AutoDock Vina software was applied to perform our primary docking protocols (60). The search space was included in a box of about 14 by 12 by $12 \AA$, with a grid spacing of $1.0 \AA$ and centered on specific cysteine residues (Cys12, 46, 66, 75, 107, 148) of GPX4, respectively. Scoring function determined by AutoDock Vina revealed that only Cys107 residues possess higher consistent binding affinity with all 3 docked compounds tested. Hence, Cys107 was selected as a starting point for subsequent covalent docking. Next, Schrödinger's Maestro suite 2015-3 was selected for performing robust covalent docking. The grid for covalent docking was identified with SiteFinder implemented in MOE (version 2015.1001; Chemical Computing Group; https://www.chemcomp.com/MOE-Molecular_Operating_Environment.htm). The grid box is defined as a spherical region encompassing Gln77, Ile106, Asp111, and His114 residues including Cys107 as reactive residue. Moreover, we predefined reaction type for each compound before proceeding with covalent docking: Michael addition and nucleophilic addition for WA, alkylation for altretamine, and nucleophilic addition for ML162. To properly validate the covalent docking protocol, we changed grid to verify stringency of docking and scoring function. In addition, post-processing after covalent docking was performed by molecular mechanics energies combined with the PoissonBoltzmann or generalized Born and surface area continuum solvation (MM/PBSA and MM/GBSA) (61). Three-dimensional molecular interactions of the docked ligands in GPX4 are displayed by PyMOL; arbitrary distances from ligand reactive groups to the specific cysteines were calculated using the measurement wizard in PyMOL (PyMOL Molecular Graphics System version 1, Schrödinger LLC).

$m R N A$ bead array and data processing. Total RNA from control and WA-treated ( $1 \mu \mathrm{M}, 24$ hours) IMR-32 and SK-N-SH cells was isolated using RNeasy Mini Kit (Qiagen, 74104) according to the manufacturer's protocol. After checking of quality control of RNA on a Bio-Rad Experion electrophoresis system, 500 ng of total RNA was amplified using the Illumina TotalPrep RNA Amplification kit (Life Technologies). Briefly, RNA was reverse-transcribed using T7 oligo-dT primers, after which biotinylated cRNA was synthesized through an in vitro transcription reaction. Seven hundred fifty nanograms of amplified cRNA was hybridized to a corresponding array of a HumanHT12 beadchip (Illumina), essentially as previously described (15). Transcriptomic analysis was done by uploading Illumina bead array gene expression data into IPA (QIAGEN Inc., https:// www.qiagenbioinformatics.com/products/ingenuitypathway-analysis) and performing a core analysis. A fold change cutoff of 2 as well as an FDR of 5\% was set to identify genes whose expression was significantly differentially regulated. A heatmap was created from the genes related to oxidative pathways using the heatmap. 2 function of the gplots R package. Raw and normalized array data were uploaded to the Gene Expression Omnibus (GEO) database with accession number GSE112384.

Synthesis of WA-NP. WA was formulated in biodegradable $\mathrm{pH}-$ responsive nanoparticles by solvent displacement. WA (6 mg) and polymer (180 mg) were dissolved in $15 \mathrm{ml}$ acetone. This solution was promptly added to $30 \mathrm{ml}$ sterile PBS and mixed. The mixture was sonicated for 1 hour, acetone was slowly evaporated under reduced pressure, and sterile water was added to compensate for the evaporated volume of water. Empty-NPs were made using a similar protocol without addition of WA. All used glasswork and material were sterilized before use. Sterile solvents were used or solvent was subjected to sterile filtration before use.

Immunohistochemistry. Tumors were dissected and fixed in $4 \%$ paraformaldehyde overnight. Subsequently tissue was embedded in paraffin and cut in 4- to 5- $\mu \mathrm{m}$ sections. Afterward, sections were dewaxed and incubated in antigen retrieval solution (DAKO, s2031) at boiling temperature for 10 minutes (for caspase-3, CD45 staining) and for 2.5 hours (for Ki67 staining). After being cooled at room temperature, endogenous peroxidase activity was blocked by immersion of the slides in peroxidase-blocking buffer (PBS solution containing $0.1 \%$ hydrogen peroxide, $0.6 \%$ sodium azide) for 20 minutes at room temperature. Blocking buffer (10\% goat serum, $1 \%$ BSA in PBS) was added to the slides for 20-30 minutes at room temperature. Primary antibody diluted in blocking buffer was incubated at $4^{\circ} \mathrm{C}$ overnight or at room temperature for 1 hour. For caspase- 3 staining, slides were incubated with secondary antibody for 1 hour, and peroxidase was detected by diaminobutyric acid substrate (DAKO, K3468). Slides were counterstained with Mayer's hematoxylin for 20 seconds and were mounted. For Ki67 and CD45 staining, slides were incubated with biotinylated secondary antibody for 40 minutes. Then, slides were incubated with VECTASTAIN ELITE ABC reagent (Vector Laboratories, PK-6100) for 20 minutes, and peroxidase was detected by diaminobutyric acid substrate. Slides were counterstained with Mayer's hematoxylin for 20 seconds and were mounted. Images were captured with an Axio Scan. $\mathrm{Z} 1$ (Zeiss). Images were acquired with a $\times 20$ Plan-Apochromat $0.8 \mathrm{NA}$ dry objective using a Hitachi HV-F202SCL camera and processed with ZEN 2012 (blue edition, Zeiss) software.

TUNEL assay. TUNEL assay was performed by use of an in situ cell death detection kit (TMR-red, Roche). Tumor section slide (as described in the Immunohistochemistry section) was dewaxed and incubated with permeabilization solution (0.1\% Triton $\mathrm{X}-100$ in $0.1 \%$ sodium citrate) for 8 minutes at room temperature. Then TUNEL reaction mixture was added to the slides and incubated on the slides for 1 hour at $37^{\circ} \mathrm{C}$. The nuclei were counterstained with DAPI (Invitrogen), and the slide was mounted and scanned with an Axio Scan.Z1 (Zeiss) using a $\times 20$ PlanApochromat 0.8 NA dry objective using a Hitachi HV-F202SCL camera. Images were processed by ZEN 2012 (blue edition, Zeiss) software.

Live cell imaging. One day before the experiment, cells were seeded (30,000 SK-N-SH cells and 50,000 IMR-32 cells per well) in an 8-well $\mu$-slide (Ibidi). The next day, the medium was replaced and SytoxGreen $(10 \mathrm{nM})$ was added to medium at least 30 minutes before imaging. Cells were treated with WA or DMSO, and time series were acquired with an LSM780 confocal microscope (Zeiss) using a $\times 63$ Plan-Apochromat 1.40 NA oil-immersion objective. The pinhole was set at 1 Airy unit (the theoretical point for optimal confocal image collection). In another setup, time series were acquired with a Zeiss Spinning Disk microscope using a $\times 40$ Plan-Apochromat $1.40 \mathrm{NA}$ oil-immersion objective and captured with a Rolera electron-multiplying charge-coupled device. Images were taken at intervals of 5 minutes for 16 hours while the system was maintained at $37^{\circ} \mathrm{C}$ and $5 \% \mathrm{CO}_{2}$. Image reconstruction was performed on Image J software. 
Immunoprecipitation of biotinylated WA. IMR-32 cells were seeded at $6 \times 10^{6}$ in a $10-\mathrm{cm}$ dish. The next day, cells were treated with the triggers (WA, biotin, biotin-WA) for 1 hour. Then cells were washed with PBS and lysed in NP-40 lysis buffer ( $\mathrm{NaCl} 150 \mathrm{mM}, \mathrm{NP}-40$ 1\%, glycerol $10 \%$, Tris- $\mathrm{HCl}, \mathrm{pH} 8,10 \mathrm{mM}$ ) supplemented with EDTA-free protease inhibitor cocktail tablets (Roche Diagnostics, 11873580001) and phosphatase inhibitor cocktail tablets (Roche Diagnostics, 04906837001). Lysate was collected and transferred to vials and rotated at $4^{\circ} \mathrm{C}$ for 15 minutes. Afterward, the lysate was centrifuged at the highest speed at $4^{\circ} \mathrm{C}$ for 15 minutes. Supernatant was collected, added to streptavidin-Sepharose beads, and incubated at $4^{\circ} \mathrm{C}$ overnight. The next day, beads were washed 3 times in NP-40 lysis buffer, and the immunoprecipitated proteins were eluted by addition of $60 \mu \mathrm{l}$ of $\times 2$ Laemmli buffer to the beads. Eluted proteins were subsequently analyzed by immunoblotting.

Determination of cellular labile $\mathrm{Fe}(\mathrm{II})$ pool. Iron measurement was performed using FeRhoNox-1. Briefly, $1 \times 10^{6}$ IMR- 32 cells were seeded in a 6-well plate 1 day before the experiment. The next day, cells were collected and centrifuged at 3,000 $g$ for 5 minutes. The cells were washed with HBSS buffer and centrifuged at 3,000 $g$ for 5 minutes. Collected cells were stained with $10 \mu \mathrm{M}$ FeRhoNox-1 in HBSS for 30 minutes in a $\mathrm{CO}_{2}$ incubator. Then cells were washed with HBSS and dissolved in $300 \mu \mathrm{l}$ of HBSS containing $1.25 \mu \mathrm{M}$ SytoxBlue and were analyzed using flow cytometry.

LC-MS analysis of phospholipids. Phospholipids from tumor tissue were analyzed by liquid chromatography-mass spectrometry (LC-MS) as previously described (62). Briefly, lipids were separated on a normal phase column [Luna $3 \mu \mathrm{m}$ Silica(2) $100 \AA$, $150 \times$ $2.0 \mathrm{~mm}$, Phenomenex] at a flow rate of $0.2 \mathrm{ml} / \mathrm{min}$ using a Dionex Ultimate 3000 HPLC system. The column was maintained at $35^{\circ} \mathrm{C}$. The analysis was performed with gradient solvents (A: propanol/ hexane/water, 285:215:5 vol/vol/vol; B: propanol/hexane/water, 285:215:40 vol/vol/vol) containing $10 \mathrm{mM}$ ammonium acetate and $0.5 \%$ triethylamine. The column was eluted for 0.5 minutes isocratically at $25 \%$ solvent B; then from 0.5 to 6.5 minutes with a linear gradient of $25 \%$ to $40 \%$ solvent B, from 6.5 to 25 minutes with a linear gradient of $40 \%$ to $55 \%$ solvent B, from 25 to 38 minutes with a linear gradient of $55 \%$ to $70 \%$ solvent $\mathrm{B}$, and from 38 to 48 minutes with a linear gradient of $70 \%$ to $100 \%$ solvent B; then isocratically from 48 to 55 minutes at $100 \%$ solvent B followed by a return to initial conditions from 55 to 70 minutes from $100 \%$ to $25 \%$ solvent B. The column was then equilibrated at $25 \%$ solvent $B$ for an additional 5 minutes. All solvents were LC-MS grade.

Statistics. Tumor size data $\left(\mathrm{mm}^{3}\right)$ were analyzed as repeated measurements, modeling the correlation structure and using the residual maximum likelihood (REML) as implemented in GenStat version 17 (https://genstat.kb.vsni.co.uk/). Significance of the main and interaction effects was assessed by an $F$ test. Cell death data were analyzed as previously described (57). Briefly, cell death data were analyzed as repeated measurements, modeling the correlation structure and using the REML as implemented in GenStat version 17. Significance of the main and interaction effects was assessed by an $F$ test. Other statistical analysis was performed using GraphPad Prism 7 software. $P$ values less than 0.05 were considered significant.

Study approval. The present studies in animals were reviewed and approved by the Ethics Committee for Laboratory Animal Experiments of Ghent University, Faculty of Sciences, Ghent, Belgium.

\section{Author contributions}

BH, JPFA, BGDG, WVB, PV, and TVB conceptualized the experiments. $\mathrm{BH}$ and TVB designed the experiments. $\mathrm{BH}, \mathrm{KH}, \mathrm{WVB}$, $\mathrm{PV}, \mathrm{VEK}$, and TVB validated the experiments; $\mathrm{BH}$ and TVB performed statistical analysis. BH, BW, II, KD, SVH, CSC, GVC, BAA, FQ, YYT, HB, EM, VEK, SMC, and MLK performed experiments, analyzed and interpreted the data. BH and TVB wrote the original draft of the manuscript. PV, WVB, BGDG, PE, SF, JPFA, MC, and VEK reviewed and edited the manuscript. $\mathrm{KH}$, BGDG, TVB, DVK, VEK, WVB, and PV got the funding. PGE, SF, $\mathrm{MC}$, and WVB provided materials and resources. WVB, PV, and TVB supervised the project and experiments.

\section{Acknowledgments}

We thank Howard Fearnhead (sabbatical visiting professor, National University of Ireland, Galway) and Kathrin Weber for fruitful discussions of the manuscript, the results, and the analysis of data. We thank the Inflammation Research Center and VIB BioImaging Core for expertise and support in microscopy and Marnik Vuylsteke for statistical support. BW is a PhD student; his research is supported by UGent Special Research Fund (BOF14/GOA/019). TVB is postdoctoral assistant at Ghent University; his research is supported by the Methusalem grant of PV. Research in PV's unit is further supported by Belgian grants (Interuniversity Attraction Poles IAP 7/32, EOS 30826052 MODEL-IDI), Flemish grants (Research Foundation Flanders: FWO G.0875.11, FWO G.0973.11, FWO G.0A45.12N, FWO G.0787.13N, FWO G.0C31.14N, FWO G.0E04.16N, G.0C76.18N, G.0B71.18N; Methusalem grants, BOF09/01M00709 and BOF16/MET_V/007), grants from Ghent University (Multidisciplinary Research Partnerships, GROUPID consortium), the Foundation against Cancer (F94/2012-188 and FAF-F/2016/865), and VIB. The research was part of the Cancer Research Institute Ghent (CRIG) network at the Ghent University. Research in WVB's unit is supported by COST Action CM1406 - Epigenetic Chemical Biology (EPICHEM) and Flemish grants (Research Foundation Flanders: FWO G.079614N and G.059713N) and a BOF(09/DOS/022) grant from Ghent University. LC-MS studies were supported by NIH NS076511, NS061817, U19 AI068021, P01 HL114453, ES020693, and CA165065. PGE is supported by the Children's Cancer Foundation and the Steven Walter Children's Cancer Foundation. We thank Anne Schepers (Laboratory of Cancer Research and Clinical Oncology, Center for Medical Genetics, University of Antwerp) for running the Illumina mRNA bead arrays. JPFA is supported by the Junior Group Leader program from the Rudolf Virchow Center, University of Würzburg.

Address correspondence to: Wim Vanden Berghe, Proteomics and Epigenetic Signalling (PPES), Department of Biomedical Sciences, University of Antwerp, Universiteitsplein 1, 2610 Wilrijk, Belgium. Phone: 3232652657; Email: Wim.vandenberghe@uantwerpen. be. Or to: Tom Vanden Berghe or Peter Vandenabeele, VIB Center for Inflammation Research, Ghent, Belgium. Technologiepark 927, 9052Zwijnaarde, Belgium. Phone: 3293313721; Email: Tom.VandenBerghe@irc.vib-ugent.be (TVB). Phone: 3293313710; Email: Peter. Vandenabeele@irc.vib-ugent.be (PV). 
VEK's present address is: Laboratory of Navigational Redox Lipidomics, IM Sechenov First Moscow State Medical University, Russia.
WVB's present address is: Laboratory of Eukaryotic Gene Expression and Signal Transduction, Department of Biochemistry and Microbiology, Ghent University, Ghent, Belgium.
1. Maris JM. Recent advances in neuroblastoma. N Engl J Med. 2010;362(23):2202-2211.

2. Cheung NK, Dyer MA. Neuroblastoma: developmental biology, cancer genomics and immunotherapy. Nat Rev Cancer. 2013;13(6):397-411.

3. Holohan C, Van Schaeybroeck S, Longley DB, Johnston PG. Cancer drug resistance: an evolving paradigm. Nat Rev Cancer. 2013;13(10):714-726.

4. Aaes TL, et al. Vaccination with necroptotic cancer cells induces efficient anti-tumor immunity. Cell Rep. 2016;15(2):274-287.

5. Yatim N, et al. RIPK1 and NF- $\mathrm{kB}$ signaling in dying cells determines cross-priming of $\mathrm{CD} 8^{+} \mathrm{T}$ cells. Science. 2015;350(6258):328-334.

6. Yang WS, et al. Regulation of ferroptotic cancer cell death by GPX4. Cell. 2014;156(1-2):317-331.

7. Vanden Berghe T, Linkermann A, Jouan-Lanhouet $S$, Walczak H, Vandenabeele P. Regulated necrosis: the expanding network of non-apoptotic cell death pathways. Nat Rev Mol Cell Biol. 2014;15(2):135-147.

8. Pasparakis M, Vandenabeele P. Necroptosis and its role in inflammation. Nature. 2015;517(7534):311-320.

9. Dixon SJ, et al. Ferroptosis: an iron-dependent form of nonapoptotic cell death. Cell. 2012;149(5):1060-1072.

10. Yang WS, Kim KJ, Gaschler MM, Patel M, Shchepinov MS, Stockwell BR. Peroxidation of polyunsaturated fatty acids by lipoxygenases drives ferroptosis. Proc Natl Acad Sci U S A. 2016;113(34):E4966-E4975

11. Torti SV, Torti FM. Iron and cancer: more ore to be mined. Nat Rev Cancer. 2013;13(5):342-355.

12. Viswanathan VS, et al. Dependency of a therapyresistant state of cancer cells on a lipid peroxidase pathway. Nature. 2017;547(7664):453-457.

13. Szarc vel Szic K, et al. Pharmacological levels of Withaferin A (Withania somnifera) trigger clinically relevant anticancer effects specific to triple negative breast cancer cells. PLoS One. 2014;9(2):e87850.

14. Kunimasa K, et al. Glucose metabolism-targeted therapy and withaferin $\mathrm{A}$ are effective for epidermal growth factor receptor tyrosine kinase inhibitor-induced drug-tolerant persisters. Cancer Sci. 2017;108(7):1368-1377.

15. Heyninck K, et al. Withaferin A induces heme oxygenase (HO-1) expression in endothelial cells via activation of the Keap1/Nrf2 pathway. Biochem Pharmacol. 2016;109:48-61.

16. Vaishnavi K, et al. Differential activities of the two closely related withanolides, Withaferin A and Withanone: bioinformatics and experimental evidences. PLoS One. 2012;7(9):e44419.

17. Rai M, Jogee PS, Agarkar G, dos Santos CA. Anticancer activities of Withania somnifera: current research, formulations, and future perspectives. Pharm Biol. 2016;54(2):189-197.

18. Vanden Berghe T, et al. Necroptosis, necrosis and secondary necrosis converge on similar cellular disintegration features. Cell Death Differ. 2010;17(6):922-930.
19. Friedmann Angeli JP, et al. Inactivation of the ferroptosis regulator $\mathrm{Gpx} 4$ triggers acute renal failure in mice. Nat Cell Biol. 2014;16(12):1180-1191.

20. Stockwell BR, et al. Ferroptosis: a regulated cell death nexus linking metabolism, redox biology, and disease. Cell. 2017;171(2):273-285.

21. Grossman EA, et al. Covalent ligand discovery against druggable hotspots targeted by anti-cancer natural products. Cell Chem Biol. 2017;24(11):1368-1376.e4.

22. Seiler A, et al. Glutathione peroxidase 4 senses and translates oxidative stress into 12/15lipoxygenase dependent- and AIF-mediated cell death. Cell Metab. 2008;8(3):237-248.

23. Sporn MB, Liby KT. NRF2 and cancer: the good, the bad and the importance of context. Nat Rev Cancer. 2012;12(8):564-571.

24. Loboda A, Damulewicz M, Pyza E, Jozkowicz A, Dulak J. Role of Nrf2/HO-1 system in development, oxidative stress response and diseases: an evolutionarily conserved mechanism. Cell Mol Life Sci. 2016;73(17):3221-3247.

25. Suttner DM, Dennery PA. Reversal of HO-1 related cytoprotection with increased expression is due to reactive iron. FASEB J. 1999;13(13):1800-1809.

26. Hirayama T, Okuda K, Nagasawa H. A highly selective turn-on fluorescent probe for iron(II) to visualize labile iron in living cells. Chem Sci. 2013;4(3):1250-1256.

27. Kagan VE, et al. Oxidized arachidonic and adrenic PEs navigate cells to ferroptosis. Nat Chem Biol. 2017;13(1):81-90.

28. Kagan VE, et al. Cytochrome $\mathrm{c}$ acts as a cardiolipin oxygenase required for release of proapoptotic factors. Nat Chem Biol. 2005;1(4):223-232.

29. Shi Y, et al. Pi-pi stacking increases the stability and loading capacity of thermosensitive polymeric micelles for chemotherapeutic drugs. Biomacromolecules. 2013;14(6):1826-1837.

30. Louage B, et al. Well-defined polymer-paclitaxel prodrugs by a grafting-from-drug approach. Angew Chem Int Ed Engl. 2016;55(39):11791-11796.

31. Shi J, Kantoff PW, Wooster R, Farokhzad OC. Cancer nanomedicine: progress, challenges and opportunities. Nat Rev Cancer. 2017;17(1):20-37.

32. Yang WS, Stockwell BR. Ferroptosis: death by lipid peroxidation. Trends Cell Biol. 2016;26(3):165-176.

33. Sun X, et al. Activation of the p62-Keap1NRF2 pathway protects against ferroptosis in hepatocellular carcinoma cells. Hepatology. 2016;63(1):173-184.

34. Fan Z, et al. Nrf2-Keap1 pathway promotes cell proliferation and diminishes ferroptosis. Oncogenesis. 2017;6(8):e371.

35. Kwon MY, Park E, Lee SJ, Chung SW. Heme oxygenase-1 accelerates erastin-induced ferroptotic cell death. Oncotarget. 2015;6(27):24393-24403.

36. Gorrini C, Harris IS, Mak TW. Modulation of oxidative stress as an anticancer strategy. Nat Rev Drug Discov. 2013;12(12):931-947.

37. Iancu TC, Shiloh H, Kedar A. Neuroblas- tomas contain iron-rich ferritin. Cancer. 1988;61(12):2497-2502.

38. Chau LY. Heme oxygenase-1: emerging target of cancer therapy. J Biomed Sci. 2015;22:22.

39. Selig RA, White L, Gramacho C, Sterling-Levis K, Fraser IW, Naidoo D. Failure of iron chelators to reduce tumor growth in human neuroblastoma xenografts. Cancer Res. 1998;58(3):473-478.

40. Tarangelo A, Dixon SJ. Nanomedicine: an iron age for cancer therapy. Nat Nanotechnol. 2016;11(11):921-922.

41. Ingold I, et al. Selenium utilization by GPX4 is required to prevent hydroperoxide-induced ferroptosis. Cell. 2018;172(3):409-422.e21.

42. Trachootham D, et al. Selective killing of oncogenically transformed cells through a ROS-mediated mechanism by beta-phenylethyl isothiocyanate. Cancer Cell. 2006;10(3):241-252.

43. Raj $\mathrm{L}$, et al. Selective killing of cancer cells by a small molecule targeting the stress response to ROS. Nature. 2011;475(7355):231-234.

44. Skouta R, et al. Ferrostatins inhibit oxidative lipid damage and cell death in diverse disease models. JAm Chem Soc. 2014;136(12):4551-4556.

45. Woo JH, et al. Elucidating compound mechanism of action by network perturbation analysis. Cell. 2015;162(2):441-451.

46. Dixon SJ, et al. Pharmacological inhibition of cystine-glutamate exchange induces endoplasmic reticulum stress and ferroptosis. Elife. 2014;3:e02523.

47. Kim SE, et al. Ultrasmall nanoparticles induce ferroptosis in nutrient-deprived cancer cells and suppress tumour growth. Nat Nanotechnol. 2016;11(11):977-985.

48. Huang Y, Dai Z, Barbacioru C, Sadée W. Cystine-glutamate transporter SLC7A11 in cancer chemosensitivity and chemoresistance. Cancer Res. 2005;65(16):7446-7454

49. Jiang L, et al. Ferroptosis as a p53-mediated activity during tumour suppression. Nature. 2015;520(7545):57-62.

50. Cramer SL, et al. Systemic depletion of L-cyst(e) ine with cyst(e)inase increases reactive oxygen species and suppresses tumor growth. Nat Med. 2017;23(1):120-127.

51. Harris IS, et al. Glutathione and thioredoxin antioxidant pathways synergize to drive cancer initiation and progression. Cancer Cell. 2015;27(2):211-222.

52. Panigrahy $\mathrm{D}$, et al. Inhibition of tumor angiogenesis by oral etoposide. Exp Ther Med. 2010;1(5):739-746.

53. Mohan R, et al. Withaferin $A$ is a potent inhibitor of angiogenesis. Angiogenesis. 2004;7(2):115-122.

54. Gupta RC, et al. Controlled-release systemic delivery - a new concept in cancer chemoprevention. Carcinogenesis. 2012;33(8):1608-1615.

55. Joshi P, Misra L, Siddique AA, Srivastava M, Kumar S, Darokar MP. Epoxide group relationship with cytotoxicity in withanolide derivatives from Withania somnifera. Steroids. 2014;79:19-27. 
56. Häcker S, et al. Histone deacetylase inhibitors cooperate with IFN-gamma to restore caspase-8 expression and overcome TRAIL resistance in cancers with silencing of caspase-8. Oncogene. 2009;28(35):3097-3110.

57. Grootjans S, et al. A real-time fluorometric method for the simultaneous detection of cell death type and rate. Nat Protoc. 2016;11(8):1444-1454.

58. Scheerer P, et al. Structural basis for cata- lytic activity and enzyme polymerization of phospholipid hydroperoxide glutathione peroxidase-4 (GPx4). Biochemistry. 2007;46(31):9041-9049.

59. Banks JL, et al. Integrated modeling program, applied chemical theory (IMPACT). JComput Chem. 2005;26(16):1752-1780.

60. Trott O, Olson AJ. AutoDock Vina: improving the speed and accuracy of docking with a new scor- ing function, efficient optimization, and multithreading. JComput Chem. 2010;31(2):455-461.

61. Li J, Abel R, Zhu K, Cao Y, Zhao S, Friesner RA. The VSGB 2.0 model: a next generation energy model for high resolution protein structure modeling. Proteins. 2011;79(10):2794-2812.

62. Tyurina YY, et al. A mitochondrial pathway for biosynthesis of lipid mediators. Nat Chem. 2014;6(6):542-552. 